\title{
Structure and dynamics of SARS-CoV-2 proofreading exoribonuclease ExoN
}

3 Nicholas H. Moeller ${ }^{1,2,3 \dagger}$, Ke Shi ${ }^{1,2,3 \dagger}$, Özlem Demir ${ }^{4 \dagger}$, Surajit Banerjee ${ }^{5}$, Lulu Yin ${ }^{1,2,3}$, Christopher

4 Belica $^{1,2,3}$, Cameron Durfee ${ }^{1,2,3}$, Rommie E. Amaro ${ }^{4}$, Hideki Aihara ${ }^{1,2,3 *}$

$6 \quad{ }^{1}$ Department of Biochemistry, Molecular Biology and Biophysics, University of Minnesota,

7 Minneapolis, Minnesota, 55455, USA

8 2Institute for Molecular Virology, University of Minnesota, Minneapolis, Minnesota, 55455, USA

$9 \quad{ }^{3}$ Masonic Cancer Center, University of Minnesota, Minneapolis, Minnesota, 55455, USA

$10{ }^{4}$ Department of Chemistry and Biochemistry, University of California, San Diego, La Jolla, CA 92093,

11 USA

$12{ }^{5}$ Northeastern Collaborative Access Team, Cornell University, Advanced Photon Source, Lemont, IL

13 60439, USA

$14 \dagger^{\dagger}$ Co-first authors

$15 *$ Correspondence: aihar001@umn.edu

\section{Abstract}

18 High-fidelity replication of the large RNA genome of coronaviruses (CoVs) is mediated by a 3'-to-5' exoribonuclease (ExoN) in non-structural protein 14 (nsp14), which excises nucleotides including antiviral drugs mis-incorporated by the low-fidelity viral RNA-dependent RNA polymerase (RdRp) and

21 has also been implicated in viral RNA recombination and resistance to innate immunity. Here we

22 determined a 1.6- $\AA$ resolution crystal structure of SARS-CoV-2 ExoN in complex with its essential co-

23 factor, nsp10. The structure shows a highly basic and concave surface flanking the active site,

24 comprising several Lys residues of nsp14 and the N-terminal amino group of nsp10. Modeling suggests

25 that this basic patch binds to the template strand of double-stranded RNA substrates to position the 3' 
end of the nascent strand in the ExoN active site, which is corroborated by mutational and computational

analyses. Molecular dynamics simulations further show remarkable flexibility of multi-domain nsp14

and suggest that nsp10 stabilizes ExoN for substrate RNA-binding to support its exoribonuclease

activity. Our high-resolution structure of the SARS-CoV-2 ExoN-nsp10 complex serves as a platform

for future development of anti-coronaviral drugs or strategies to attenuate the viral virulence.

\section{Introduction}

The $29.9 \mathrm{~kb}$ single-stranded RNA genome of SARS-CoV-2, the causative agent of the global COVID-19

(1-3). Unlike the high-fidelity cellular replicative DNA polymerases, viral RdRp enzymes including the

coronavirus $(\mathrm{CoV}) \mathrm{RdRp}$ do not contain a proofreading exonuclease domain to ensure high fidelity. The

resulting higher mutation rate $\left(10^{-4}\right.$ to $10^{-6}$ substitutions/nucleotide/round of replication) is generally

thought to promote rapid viral adaptation in response to selective pressure (4-6). However, the lack of

proofreading activity in RdRp poses a particular challenge for the replication of coronaviruses, which

feature the largest known RNA virus genomes $(27 \sim 32 \mathrm{~kb}$, up to twice the length as the next-largest

non-segmented RNA viral genomes) $(7,8)$. It has been reported that SARS-CoV nsp12 is the fastest

viral RdRp known but with an error rate more than one order of magnitude higher than the generally

admitted error rate of viral RdRps (9), clearly necessitating a unique proofreading mechanism.

To mitigate the low fidelity of RdRp, all coronaviruses encode a 3'-to-5' exoribonuclease (ExoN)

in nsp14 (10-12). Mutations of SARS-CoV-2 nsp14 exhibit strong association with increased genome-

wide mutation load $(13,14)$, and genetic inactivation of ExoN in engineered SARS-CoV and murine

hepatitis virus (MHV) leads to 15 to 20 -fold increases in mutation rates $(7,15,16)$. Furthermore, in a

mouse model, SARS-CoV with inactivated ExoN shows a mutator phenotype with decreased fitness and

lower virulence over serial passage, suggesting a potential strategy for generating a live, impaired- 
SARS-CoV-2 and Middle East Respiratory Syndrome (MERS)-CoV (18), hinting at additional functions

RNA recombination required for subgenomic mRNA synthesis during normal replication of CoVs

including SARS-CoV-2 (19), and it was shown to be required for resistance to the antiviral innate

immune response for MHV (20). ExoN inactivation also significantly increases the sensitivity of CoVs

to nucleoside analogs that target RdRp, which is consistent with the biochemical activity of ExoN to

excise mutagenic or chain-terminating nucleotides mis-incorporated by $\operatorname{RdRp}(21-23)$. These

observations combine to suggest that chemical inhibition of ExoN could be an effective antiviral

strategy against CoVs. In this study, we determined a high-resolution crystal structure of the SARS-

CoV-2 ExoN-nsp10 complex and studied its biochemical activities. Furthermore, we used molecular

61 dynamics (MD) simulations to better understand the dynamics of nsp14, nsp10, and their interaction with RNA.

\section{Results}

The multifunctional SARS-CoV-2 nsp14 consists of the N-terminal ExoN domain involved in proofreading and the C-terminal guanine N7 methyl transferase (N7-MTase) domain that functions in mRNA capping. We co-expressed in bacteria the full-length 527-residue SARS-CoV-2 nsp14 or its Nterminal fragment (residues 1 to 289) containing only the ExoN domain, with full-length 139-residue nsp10 in both cases and purified the heterodimeric complexes. The nsp14-nsp10 and ExoN-nsp10 complexes both showed the expected 3'-to-5' exonuclease activity on a 5'-fluorescently labeled 20nucleotide (nt) RNA (LS2U: 5'-GUCAUUCUCCUAAGAAGCUU; similar to 'LS2' used previously in SARS-CoV ExoN studies (21)) (Fig. 1A, B). Although LS2U RNA by itself served as a substrate, more extensive degradation was observed when it was annealed to an unlabeled 40-nt template strand 
(LS15_RNA; Table 1) had no discernable effect on the processing by either complex (Fig. 1A, B).

When DNA was used as the template strand (LS15_DNA; Table 1) to generate an RNA/DNA

heteroduplex substrate that is expected to take the A-form conformation similarly to dsRNA, the activity was observed but weaker than for dsRNA. No nuclease activity was observed on a 5'-fluorescently labeled 20-nt DNA (LS2_DNA; Table 1), whether the template strand was RNA (LS15_RNA), DNA (LS15_DNA; Table 1), or absent. A 20-nt poly-U RNA (U20_RNA; Table 1), which is less likely to adopt secondary structures than LS2U, did not serve as a substrate by itself but was degraded extensively when supplemented with a complementary 30-nt poly-A RNA (A30_RNA; Table 1) (Fig. 1C). Collectively, these results show that the N-terminal ExoN domain of SARS-CoV-2 nsp14 is sufficient for binding to nsp10 to form an active exoribonuclease complex that preferentially degrades dsRNA. For comparison, we also generated a corresponding SARS-CoV ExoN-nsp10 complex, which showed similar activities to SARS-CoV-2 ExoN-nsp10 (Fig. 1C, Supplementary Fig. 1).

Previous X-ray crystallographic studies have provided the structure of SARS-CoV nsp14-nsp10 complex at resolutions ranging from 3.2 to $3.4 \AA(21,24)$. To obtain higher resolution view of a CoV exoribonuclease complex and to reveal possible structural difference between SARS-CoV and SARSCoV-2 ExoN, we have crystallized the SARS-CoV-2 ExoN-nsp10 complex. An ExoN variant with a nuclease-inactivating mutation (E191Q) (Fig. 1C, Supplementary Fig. 1) was used in our crystallographic studies as it was expressed more robustly and generated a more stable complex with nsp10 than wild-type ExoN. We obtained crystals under two different conditions, one containing ammonium tartrate and the other containing magnesium chloride $\left(\mathrm{MgCl}_{2}\right)$, albeit in the same crystal form. The structures were determined by molecular replacement phasing and refined to 1.64 and $2.10-\AA$ resolution for the tartrate and magnesium-bound crystals, respectively (Fig. 2A, Table 2). The final models consist of nsp14 residues Asn3 to Arg289 (Val287 for the lower resolution structure) and nsp10 residues Ala1 to Cys130, with two zinc ions bound to each polypeptide chain. As expected from high sequence conservations, SARS-CoV-2 ExoN-nsp10 complex shows high structural similarity to its 
101 counterpart from SARS-CoV (root-mean-square deviation of $0.95 \AA$ for all main chain atoms against

102 5C8T (24)), whose shape was previously described to resemble 'hand (ExoN) over a fist (nsp10)' (21)

103 (Fig. 2B). A superposition between the SARS-CoV and SARS-CoV-2 ExoN-nsp10 structures shows

104 only relatively small (3.0 $\AA$ or less) deviations in several regions of the complex, including the tip of the

105 'fingers' region of ExoN comprising nsp14 residues $40 \sim 50$, and surface-exposed loops of nsp10

106 (Supplementary Fig. 2).

While our structures of SARS-CoV-2 ExoN-nsp10 obtained in the two different crystallization conditions are highly similar to each other, they show notable differences in the exonuclease active site located around the 'knuckles' of ExoN. In the crystal grown in the presence of $\mathrm{MgCl}_{2}$, we observed a magnesium ion octahedrally coordinated by Asp90, Glu92, Asp273, and three water molecules (Fig. 2C,

111 Supplementary Fig. 3d). Another magnesium ion required for the conserved two-metal ion mechanism

112 of 3'-5' editing exonucleases $(25,26)$ was not observed. The previously reported SARS-CoV nsp14-

113 nsp10 structures also showed only one metal ion, bound at an alternative site between Asp90 and

114 Glu191 $(21,24)$. This site is unoccupied in our structure presumably due to the E191Q mutation. In

115 contrast, the higher resolution tartrate-bound structure shows a unique configuration of metal-free active

116 site (Fig. 2D, Supplementary Fig. 3c). Without the magnesium ion, Asp90 takes two distinct

117 conformers with its carboxylate group in orthogonal orientations. Glu92 is pointed away from

118 Asp90/Asp273 and hydrogen-bonded to Gln108 side chain, whereas His268 in turn is flipped away from

119 Glu92. A comparison between the $\mathrm{Mg}^{2+}$-bound and free structures shows a significant rearrangement for 120 residues Gly265 to Val269 including the main chain atoms, accompanying an inward movement of

121 His268 upon $\mathrm{Mg}^{2+}$-binding (Fig. 2E). These observations demonstrate high flexibility of the ExoN

122 active site in the absence of divalent metal co-factors.

To obtain an idea about how SARS-CoV-2 ExoN-nsp10 complex engages RNA substrates, we

124 modeled an RNA-bound ExoN-nsp10 structure based on the double-stranded (ds) RNA-bound structures

125 of Lassa virus nucleoprotein (NP) exonuclease domain, which is another DEDDh-family $3^{\prime}$-to-5' 
exoribonuclease. A superposition of the Lassa NP-RNA complex $(27,28)$ on ExoN-nsp10 based on their conserved catalytic residues (Lassa NP: D389/E391/D466/H528/D533 according to the numbering in 4FVU (27), vs. SARS-CoV-2 ExoN: D90/E92/E191/H268/D273) places the A-form dsRNA in a shallow groove on ExoN surface adjacent to the active site, with remarkable shape complementarity

(Fig. 3 B, C). In this model, the sugar-phosphate backbone of the non-degradable (template) RNA

131 strand tracks a positively charged patch on the ExoN surface including Lys9 and Lys61, whereas the 3'

132 end of its complementary (degradable) strand is presented to the active site. The extensive protein 133 contacts made by the non-degradable strand in a dsRNA substrate is consistent with the preference for 134 dsRNA substrates by SARS-CoV-2 ExoN as shown above (Fig. 1) and by SARS-CoV ExoN reported 135 earlier (29). Notably, we observed ordered tartrate ions from the crystallization condition bound to this 136 basic patch in our crystal structure, potentially mimicking RNA backbone phosphate interactions 137 (Supplementary Figs. 3a, 3b, and 4). co-expressed with nsp10 and purified as heterodimeric complexes. In the exoribonuclease assay using

144 the RNA substrates described above, all three lysine-to-alanine mutants showed lower activity than 145 wild-type ExoN (Fig. 4). In particular, the K9A and K61A substitutions caused severer defect than 146 K139A, consistent with our dsRNA-binding model (Fig. 3 B, C). While the precise conformation of 147 LS2U RNA in the absence of a complementary strand is unknown, its binding to ExoN must also 148 depend on these Lys residues, underscoring the importance of electrostatic interactions with RNA by the 149 mutated lysine residues in the ExoN activity. 
Previous studies showed that the exoribonuclease activity of nsp14 is strongly stimulated by

nsp10 for both SARS-CoV and SARS-CoV-2 (29-32). In our crystal structure, the N-terminal residues

of ExoN and those of nsp10 are wrapped around each other in a 'criss-cross' arrangement and forming

Fig. 3a). In addition, the first $\alpha$-helix of nsp10 interacts with the ExoN loop harboring nsp14 Lys61,

3A). In the absence of nsp10 supporting the RNA-binding groove from the back (Fig. 3C,

Supplementary Figs. 5, 6), the N-terminal residues of ExoN including nsp14 Lys9 and those around

Lys61 are likely to be more flexible. Moreover, the terminal amino group of nsp10 Ala1 is part of the basic patch and involved in direct RNA backbone contact in our protein-RNA docking model (Fig. 3B,

Supplementary Fig. 5). These observations may together explain the strong stimulation of ExoN

161 activity by nsp10.

To obtain further insights into the role of nsp10 and to support our RNA-binding model, we

CoV-2 nsp14, constructed from our ExoN-nsp10 co-crystal structure and a homology model of the Csimulations (GAMD) totaling 0.6- $\mu$ s were performed for each system to enhance conformational

171 (nsp14 residues 1-60), which showed large deviations from the starting model and eventually became

172 highly disordered in the absence of nsp10. A principal component analysis for the 3 systems show that

173 the conformational space sampled by nsp14 is significantly larger in the absence of nsp10 (Fig. 5A,

\section{Supplementary Fig. 7).}


The first principal component (PC1), which is broadly sampled by all 3 systems, corresponds to

Fig. 8, Supplementary animation 1). In the conformation with minimal PC1 (Fig. 5B, left), the substrates (S-adenosyl methionine [SAM] and GpppA)-binding cleft of the N7-MTase domain abuts against the ExoN domain, leading to occlusion of the substrates. On the other extreme with maximal

$180 \mathrm{PC} 1$, the cleft is more open to the solvent (Fig. 5B, right). The second principal component (PC2)

181 corresponds to an ordered-to-disordered transition of the 'fingers' region of ExoN, which shows a large 182 population of disordered conformations only for the nsp14-alone system as mentioned above (Fig. 5C,

183 Supplementary Fig. 9, Supplementary animations 2, 3). Although folding of the core of the ExoN

184 domain does not depend on nsp10, residues Lys9 and Lys61 important for RNA-binding and the 185 surrounding residues show increased flexibility in the absence of nsp10, confirming our prediction above (Table 3, Fig. 5 D, E, Supplementary Fig. 9, Supplementary animation 3). The dsRNA molecule in the nsp14-nsp10-RNA complex was stable throughout the simulation with direct RNA phosphate contacts by nsp14 Lys9, Lys61, and the terminal amino group of nsp10 maintained, providing

189 further support for our model for dsRNA-binding (Fig. 5 F, G, Supplementary Fig. 10). An ionic 190 interaction between Ala1 of nsp10 and RNA backbone phosphate was particularly persistent and 191 observed for $97 \%$ of the time during the simulations (3.2 $\AA$ distance cutoff), which led to a significant 192 stabilization of this residue in the presence of RNA (Table 3). Lastly, it is also worth noting that an 193 analysis of the internal dynamics of the N7-MTase domain indicates several highly mobile regions, 194 including loops (residues 289-300, 355-362) flanking the substrates-binding cleft and a loop (residues 195 454-470) adjacent to the third zinc finger motif of nsp14 distal to the N7-MTase active site

\section{6 (Supplementary Fig. 11).}

\section{Discussion}


ExoN-nsp10 complex preferentially degrades dsRNA substrates. This is in contrast to the proofreading

exonuclease domain of high-fidelity DNA polymerases, whose active site engages the single-stranded

DNA 3 ' end in partially melted double-stranded substrates $(25,33)$, and suggests a unique mechanism of proofreading. The extensive ExoN/nsp10 interface buries a total of $2203 \AA^{2}$ of surfaces from both proteins, spanning both the 'fingers' and 'palm' regions of ExoN. Folding of the fingers region depends on its interaction with nsp10, which involves several critical residues including nsp10 Tyr96 (31) (Fig.

2A, Supplementary Fig. 6). On the other hand, an interesting feature for the interaction in the palm region includes the insertion of Phe16 and Phe19 from the first $\alpha$-helix of nsp10 into a deep hydrophobic pocket of ExoN, which is essential for the stable complex formation (31). Notably, this hydrophobic pocket is located on the backside from the ExoN active site, where nsp10 Phe19 side chain makes van der Waals contacts with the main chain of an ExoN $\alpha$-helix harboring one of the catalytic residues Glu191 (Supplementary Fig. 6). Thus, targeting said pocket of ExoN by small molecules to block its interaction with nsp10 or potentially to allosterically modulate its catalytic activity could be a possible strategy of inhibition.

MD simulations revealed remarkable flexibility in full-length nsp14 (Supplementary Figs. 7, 8, and Supplementary animations 1, 2), which affects solvent accessibility of the SAM/GpppA-binding cleft and may play an important role in the catalytic cycle of N7-MTase (Fig. 5B). Similar conformational variation, albeit with a much smaller magnitude, was previously observed between two SARS-CoV nsp14 molecules in the asymmetric unit of a crystal (Supplementary Fig. 8) (21). Although this hinge motion was observed for all 3 systems (nsp14-alone, nsp14-nsp10, and nsp14-nsp10-RNA) in 
combinations of PC1 and PC2 values (Fig. 5A, left), suggesting that there may be a long-range

interaction between the N-terminal fingers region of ExoN and the C-terminal N7-MTase domain. These

observations are consistent with earlier studies showing that single amino acid substitutions R84A and

W86A within the ExoN domain completely abolished, while a deletion of the N-terminal 61 residues

significantly enhanced, the N7-MTase activity of SARS-CoV nsp14 (34). These mutations in ExoN may

have modulated the PC1 motion of nsp14 to affect its N7-MTase activity. Conversely, although we

showed in this study that the N7-MTase domain is not essential for the exoribonuclease activity of nsp14

in vitro, SARS-CoV nsp14 N7-MTase residues Tyr498 and His487 were shown to be required for

$\mathrm{RdRp} / \mathrm{nsp} 12$ binding (21), which is presumably important in proofreading. Thus, it is likely that the

ExoN and N7-MTase domains are functionally dependent on each other, where proper dynamics may be

key to support their respective activities and possible coordination. We hope that our structural and

$\mathrm{CoV}-2$ and related coronaviruses.

\section{Methods}

Protein expression and purification

SARS-CoV-2 (GenBank: MN908947.3) nsp14 and nsp14(1-289) were co-expressed with nsp10 in $E$.

coli strain BL21(DE3) under the control of T7 promoters. To facilitate purification, a 6xHis tag was 
1 hour at $4{ }^{\circ} \mathrm{C}$, after which the protein complex in the supernatant was captured by nickel-affinity

$3 \mathrm{C}$ protease overnight at $4{ }^{\circ} \mathrm{C}$, concentrated by ultrafiltration, and passed through a Superdex 75 size-

exclusion column operating with the same buffer as above except not containing imidazole. The nsp14-

nsp10 complexes eluted as a heterodimer were concentrated by ultrafiltration and frozen in small

volume aliquots in liquid nitrogen for storage at $-80^{\circ} \mathrm{C}$. The ExoN mutant derivatives were generated by

site-directed mutagenesis and purified using the same procedure. The protein concentrations were

determined based on UV absorbance at $280 \mathrm{~nm}$ measured on a Nanodrop 8000 spectrophotometer and theoretical extinction coefficients calculated from the protein amino acid sequences.

\section{Crystallization and structure determination}

260 Purified nsp14(1-289, E191Q)-nsp10 complex (Supplementary Fig. 12) at $17 \mathrm{mg} \mathrm{ml}^{-1}$ was crystallized 261 using the hanging drop vapor diffusion method, by mixing the protein solution with an equal volume of reservoir solution including either $0.2 \mathrm{M}$ di-ammonium tartrate, $\mathrm{pH}$ 7.0, $20 \%$ polyethylene glycol (PEG)

conditions produced thin needles crystals. The crystals were cryo-protected with ethylene glycol and molecular replacement phasing by PHASER (36), using the crystal structures of SARS-CoV nsp14nsp10 complex (PDB ID: 5C8T) (24) as the search model. Iterative model building and refinement were performed using COOT (37) and PHENIX (38), respectively. A summary of data collection and model

271 refinement statistics is shown in Table 2. Structure images were generated using PyMOL

272 (https://pymol.org/). 
Exonuclease activity assays

275 The 5'-fluorescein labeled oligonucleotides (Table 1) at $750 \mathrm{nM}$, in the presence or absence of

276 equimolar complementary unlabeled strands, were incubated with $50 \mathrm{nM}$ nsp14 (or its ExoN domain

277 alone)-nsp10 complexes in $42 \mathrm{mM}$ Tris- $\mathrm{HCl}, \mathrm{pH} 8.0,0.94 \mathrm{mM} \mathrm{MgCl}, 0.94 \mathrm{mM}$ dithiothreitol, and

$2780.009 \%$ Tween-20. After incubation at $37{ }^{\circ} \mathrm{C}$ for $10 \mathrm{~min}$, the reactions were stopped by the addition of

279 formamide to $67 \%$ and heating to $95^{\circ} \mathrm{C}$ for $10 \mathrm{~min}$. The reaction products were separated on a $15 \%$

280 TBE-Urea gel, which was scanned on a Typhoon FLA 9500 imager.

\section{Molecular dynamics simulations}

283 A homology model of full-length SARS-CoV-2 nsp14 was generated for sequence of YP_009725309.1

and taking SARS-CoV nsp14 crystal structure (PDB ID: 5NFY) (21) as a template in Schrödinger Prime

module (39). The nsp14 ExoN domain of the homology model was then replaced with the crystal

structure of SARS-CoV-2 nsp14 ExoN in complex with nsp10 obtained in this study. E191Q mutation

in the crystal structure was reverted computationally to the wild type. For a nsp14-nsp10-RNA model,

RNA was modeled based on Lassa NP-RNA complex (PDB ID: 4FVU) (27) and the second Mg ion at

the active site was modeled based on a $\mathrm{Mn}^{2+}$ ion found in Lassa NP-RNA complex (PDB ID: 4GV9)

(28). Three systems were prepared from this model: 1. Full-length nsp14 alone, 2. Full-length nsp14-

nsp10 complex, 3. Full-length nsp14-nsp10-RNA complex. Protonation states of titratable amino acids

were determined using PropKa analysis (40). Each of these systems were explicitly solvated in TIP3P water box and ions were added to achieve 0.2 M salt concentration. Amber ff14SB (41) and RNA.OL3

294 force fields are used for protein and RNA, respectively. For zinc ions and zinc-coordinating residues,

295 Cationic Dummy Atom (CADA) parameters were used (42). Conventional MD simulations (cMD) were 296 performed with NAMD2.14 program (43), while Gaussian-accelerated MD simulations (GAMD) were 297 performed with Amber20 program (44). First, each system was minimized in 4 consecutive steps by 298 gradually decreasing restraints. Subsequently, each system was heated from 0 to $310 \mathrm{~K}$ slowly, and then 
299 equilibrated for about 1 ns by gradually decreasing restraints in 3 consecutive steps. For cMD, three

300 independent copies $(2 \mathrm{x} 1 \mu \mathrm{s}$ and $1 \times 0.6 \mu \mathrm{s})$ of simulation were run for each system. For GAMD, three

301 independent copies of $0.2 \mu$ s of simulation were run for each system using dual boost method following

302 a 20-ns MD run to calculate parameters for GAMD production runs. All cMD and GAMD simulations

303 were performed at $310 \mathrm{~K}$ and 1 atm and with a $2 \mathrm{fs}$ timestep. For each system, 32,000 data points with

$3040.1 \mathrm{~ns}$ intervals were collected from simulations and analyzed. Stability of MD simulations are shown

305 with RMSD plots of nsp14 ExoN domain (Supplementary Fig. 13). MDTraj (45) was used for some of 306 the MD trajectory analysis.

\section{Acknowledgements}

309 We thank Daniel Harki and Reuben Harris for thoughtful comments. This work was supported by grants 310 from the US National Institutes of Health (NIGMS R35-GM118047 to H.A., R01-GM132826 to R.E.A., 311 and NCI P01-CA234228 to R.E.A. and H.A.), NSF RAPID MCB-2032054, an award from the RCSA

312 Research Corp., and a UC San Diego Moores Cancer Center 2020 SARS-COV-2 seed grant to R.E.A.

313 This work is based upon research conducted at the Northeastern Collaborative Access Team beamlines,

314 which are funded by the US National Institutes of Health (NIGMS P30 GM124165). The Pilatus 6M

315 detector on 24-ID-C beamline is funded by a NIH-ORIP HEI grant (S10 RR029205). This research used

316 resources of the Advanced Photon Source, a U.S. Department of Energy (DOE) Office of Science User

317 Facility operated for the DOE Office of Science by Argonne National Laboratory under Contract No.

318 DE-AC02-06CH11357 and those of the Minnesota Supercomputing Institute. We are grateful for the

319 efforts of the Texas Advanced Computing Center (TACC) Frontera team and for the compute time made

320 available through a Director's Discretionary Allocation (made possible by the National Science

321 Foundation award OAC-1818253). 
bioRxiv preprint doi: https://doi.org/10.1101/2021.04.02.438274; this version posted April 4, 2021. The copyright holder for this preprint (which was not certified by peer review) is the author/funder, who has granted bioRxiv a license to display the preprint in perpetuity. It is made available under aCC-BY-NC-ND 4.0 International license.

324 The atomic coordinates and structure factors for the SARS-CoV-2 ExoN-nsp10 complex structures have

325 been deposited in the RCSB Protein Data Bank, with the accession codes 7MC5 and 7MC6.

\section{Competing Interests}

328 The authors have no competing interests to declare.

\section{Reference:}

331 1. Hillen HS, et al. (2020) Structure of replicating SARS-CoV-2 polymerase. Nature 584(7819):154156.

2. $\quad$ Posthuma CC, Te Velthuis AJW, \& Snijder EJ (2017) Nidovirus RNA polymerases: Complex enzymes handling exceptional RNA genomes. Virus Res 234:58-73. Sola I, Almazan F, Zuniga S, \& Enjuanes L (2015) Continuous and Discontinuous RNA Synthesis in Coronaviruses. Annu Rev Virol 2(1):265-288.

5. Jenkins GM, Rambaut A, Pybus OG, \& Holmes EC (2002) Rates of molecular evolution in RNA viruses: a quantitative phylogenetic analysis. J Mol Evol 54(2):156-165.

6. Sanjuan R, Nebot MR, Chirico N, Mansky LM, \& Belshaw R (2010) Viral mutation rates. J Virol 84(19):9733-9748.

7. Denison MR, Graham RL, Donaldson EF, Eckerle LD, \& Baric RS (2011) Coronaviruses: an RNA proofreading machine regulates replication fidelity and diversity. RNA Biol 8(2):270-279.

8. Gorbalenya AE, Enjuanes L, Ziebuhr J, \& Snijder EJ (2006) Nidovirales: evolving the largest RNA virus genome. Virus Res 117(1):17-37.

9. Shannon A, et al. (2020) Rapid incorporation of Favipiravir by the fast and permissive viral RNA polymerase complex results in SARS-CoV-2 lethal mutagenesis. Nat Commun 11(1):4682.

10. Minskaia E, et al. (2006) Discovery of an RNA virus 3'->5' exoribonuclease that is critically involved in coronavirus RNA synthesis. Proc Natl Acad Sci U S A 103(13):5108-5113.

11. Robson F, et al. (2020) Coronavirus RNA Proofreading: Molecular Basis and Therapeutic Targeting. Mol Cell 79(5):710-727.

12. Smith EC \& Denison MR (2013) Coronaviruses as DNA wannabes: a new model for the regulation of RNA virus replication fidelity. PLoS Pathog 9(12):e1003760.

13. Eskier D, Suner A, Oktay Y, \& Karakulah G (2020) Mutations of SARS-CoV-2 nsp14 exhibit strong association with increased genome-wide mutation load. PeerJ 8:e10181.

14. Takada K, Takahashi Ueda M, Watanabe T, \& Nakagawa S (2020) Genomic diversity of SARSCoV-2 can be accelerated by a mutation in the nsp14 gene. bioRxiv 2020.12.23.424231; doi: https://doi.org/10.1101/2020.12.23.424231

15. Eckerle LD, et al. (2010) Infidelity of SARS-CoV Nsp14-exonuclease mutant virus replication is revealed by complete genome sequencing. PLoS Pathog 6(5):e1000896.

16. Eckerle LD, Lu X, Sperry SM, Choi L, \& Denison MR (2007) High fidelity of murine hepatitis virus replication is decreased in nsp14 exoribonuclease mutants. J Virol 81(22):12135-12144. 
364 17. Graham RL, et al. (2012) A live, impaired-fidelity coronavirus vaccine protects in an aged, immunocompromised mouse model of lethal disease. Nat Med 18(12):1820-1826.

18. Ogando NS, et al. (2020) The Enzymatic Activity of the nsp14 Exoribonuclease Is Critical for Replication of MERS-CoV and SARS-CoV-2. J Virol 94(23).

19. Gribble J, et al. (2021) The coronavirus proofreading exoribonuclease mediates extensive viral recombination. PLoS Pathog 17(1):e1009226.

20. Case JB, et al. (2018) Murine Hepatitis Virus nsp14 Exoribonuclease Activity Is Required for Resistance to Innate Immunity. J Virol 92(1).

21. Ferron F, et al. (2018) Structural and molecular basis of mismatch correction and ribavirin excision from coronavirus RNA. Proc Natl Acad Sci U S A 115(2):E162-E171.

22. Agostini ML, et al. (2018) Coronavirus Susceptibility to the Antiviral Remdesivir (GS-5734) Is Mediated by the Viral Polymerase and the Proofreading Exoribonuclease. mBio 9(2).

23. Smith EC, Blanc H, Surdel MC, Vignuzzi M, \& Denison MR (2013) Coronaviruses lacking exoribonuclease activity are susceptible to lethal mutagenesis: evidence for proofreading and potential therapeutics. PLoS Pathog 9(8):e1003565.

24. Ma Y, et al. (2015) Structural basis and functional analysis of the SARS coronavirus nsp14-nsp10 complex. Proc Natl Acad Sci U S A 112(30):9436-9441.

25. Beese LS \& Steitz TA (1991) Structural basis for the 3'-5' exonuclease activity of Escherichia coli DNA polymerase I: a two metal ion mechanism. EMBO J 10(1):25-33.

26. Chen P, et al. (2007) Biochemical characterization of exoribonuclease encoded by SARS coronavirus. J Biochem Mol Biol 40(5):649-655.

27. Hastie KM, King LB, Zandonatti MA, \& Saphire EO (2012) Structural basis for the dsRNA specificity of the Lassa virus NP exonuclease. PLoS One 7(8):e44211.

28. Jiang $X$, et al. (2013) Structures of arenaviral nucleoproteins with triphosphate dsRNA reveal a unique mechanism of immune suppression. J Biol Chem 288(23):16949-16959.

29. Bouvet $\mathrm{M}$, et al. (2012) RNA 3'-end mismatch excision by the severe acute respiratory syndrome coronavirus nonstructural protein nsp10/nsp14 exoribonuclease complex. Proc Natl Acad Sci U S A 109(24):9372-9377.

30. Bouvet M, et al. (2014) Coronavirus Nsp10, a critical co-factor for activation of multiple replicative enzymes. J Biol Chem 289(37):25783-25796.

31. Saramago $M$, et al. (2021) New targets for drug design: Importance of nsp14/nsp10 complex formation for the 3'-5' exoribonucleolytic activity on SARS-CoV-2. bioRxiv 2021.01.07.425745; doi: https://doi.org/10.1101/2021.01.07.425745

32. Baddock HT, et al. (2020) Characterisation of the SARS-CoV-2 ExoN (nsp14ExoN-nsp10) complex: implications for its role in viral genome stability and inhibitor identification. bioRxiv 2020.08.13.248211; doi: https://doi.org/10.1101/2020.08.13.248211.

33. Beese LS, Derbyshire V, \& Steitz TA (1993) Structure of DNA polymerase I Klenow fragment bound to duplex DNA. Science 260(5106):352-355.

34. Chen $Y$, et al. (2013) Structure-function analysis of severe acute respiratory syndrome coronavirus RNA cap guanine-N7-methyltransferase. J Virol 87(11):6296-6305.

35. Kabsch W (2010) Xds. Acta Crystallogr D Biol Crystallogr 66(Pt 2):125-132.

36. McCoy AJ, et al. (2007) Phaser crystallographic software. J App/ Crystallogr 40(Pt 4):658-674. Crystallogr D Biol Crystallogr 66(Pt 4):486-501.

38. Liebschner D, et al. (2019) Macromolecular structure determination using X-rays, neutrons and 
410 39. Schrödinger (2021) Schrödinger Release 2021-1: Prime, Schrödinger, LLC, New York, NY.

411 40. Dolinsky TJ, Nielsen JE, McCammon JA, \& Baker NA (2004) PDB2PQR: an automated pipeline for the setup of Poisson-Boltzmann electrostatics calculations. Nucleic Acids Res 32(Web Server issue):W665-667.

414 41. Maier JA, et al. (2015) ff14SB: Improving the Accuracy of Protein Side Chain and Backbone Parameters from ff99SB. J Chem Theory Comput 11(8):3696-3713.

42. Pang YP (1999) Novel Zinc Protein Molecular Dynamics Simulations: Steps Toward Antiangiogenesis for Cancer Treatment. J Mol Model 5:196-202.

43. Phillips JC, et al. (2020) Scalable molecular dynamics on CPU and GPU architectures with NAMD. J Chem Phys 153(4):044130.

44. Case DA, et al. (2020) AMBER 2020, University of California, San Francisco.

45. McGibbon RT, et al. (2015) MDTraj: A Modern Open Library for the Analysis of Molecular Dynamics Trajectories. Biophys J 109(8):1528-1532.

46. Baker NA, Sept D, Joseph S, Holst MJ, \& McCammon JA (2001) Electrostatics of nanosystems: application to microtubules and the ribosome. Proc Natl Acad Sci U S A 98(18):10037-10041. 
bioRxiv preprint doi: https://doi.org/10.1101/2021.04.02 438274 t this version posted April 4, 2021. The copyright holder for this preprint (which was not certified by peer review) is the author/funder, who has granted bioRxiv a license to display the preprint in perpetuity. It is made available under aCC-BY-NC-ND 4.0 International license.

426 Table 1: Oligonucleotides used in biochemical assays

\section{LS2U}

/56-FAM/rGrUrCrArUrUrCrUrCrCrUrArArGrArArGrCrUrU

LS15A_RNA

rCrUrArUrCrCrCrCrArUrGrUrGrArUrUrUrUrArCrArArGrCrUrUrCrUrUrArGrGrArGrArArUrGrArC

LS15_RNA

rCrUrArUrCrCrCrCrArUrGrUrGrArUrUrUrUrArCrUrArGrCrUrUrCrUrUrArGrGrArGrArArUrGrArC

LS2_DNA

/56-FAM/GTCATTCTCCTAAGAAGCTA

\section{LS15_DNA}

CTATCCCCATGTGATTTTACTAGCTTCTTAGGAGAATGAC

U20_RNA

/56-FAM/rUrUrUrUrUrUrUrUrUrUrUrUrUrUrUrUrUrUrUrU

A30_RNA

rArArArArArArArArArArArArArArArArArArArArArArArArArArArArArA

'56-FAM' denotes 5' 6-Fluorescein. ' $r$ ' denotes ribonucleotide. 
442 Table 2: Summary of X-ray data collection and model refinement statistics

\begin{tabular}{|c|c|c|}
\hline & ExoN-nsp10 (7MC5) & ExoN-nsp10-Mg ${ }^{2+}(7 \mathrm{MC} 6)$ \\
\hline \multicolumn{3}{|l|}{ Data collection } \\
\hline Wavelength $(\AA)$ & 0.979 & 0.979 \\
\hline Resolution range $(\AA)$ & $57.7-1.64(1.70-1.64)$ & $42.6-2.10(2.18-2.10)$ \\
\hline Space group & $\mathrm{P} 2{ }_{1} 2_{1} 2_{1}$ & $\mathrm{P} 2{ }_{1} 2_{1} 2_{1}$ \\
\hline Unit cell $(a, b, c$ in $\AA)$ & 63.7467 .48111 .25 & 61.6770 .32108 .54 \\
\hline Total reflections & $258196(22096)$ & $105896(10815)$ \\
\hline Unique reflections & $58702(5273)$ & $27756(2767)$ \\
\hline Multiplicity & $4.4(4.2)$ & $3.8(3.9)$ \\
\hline Completeness (\%) & $98.81(90.43)$ & 98.25 (99.43) \\
\hline$</ / \sigma(I)>$ & $12.57(1.48)$ & $10.70(1.96)$ \\
\hline$R_{\text {merge }}$ & $0.148(1.22)$ & $0.078(0.928)$ \\
\hline$R_{\text {meas }}$ & $0.166(1.40)$ & $0.091(1.082)$ \\
\hline $\mathrm{R}_{\text {p.i.m. }}$ & $0.076(0.660)$ & $0.045(0.543)$ \\
\hline $\mathrm{CC}_{1 / 2}$ & $0.995(0.394)$ & $0.997(0.524)$ \\
\hline \multicolumn{3}{|l|}{ Refinement } \\
\hline Reflections, working set & $58626(5273)$ & $27755(2768)$ \\
\hline Reflections, test set & $2826(251)$ & $1364(132)$ \\
\hline$R_{\text {work }}$ & $0.166(0.354)$ & $0.197(0.306)$ \\
\hline$R_{\text {free }}$ & $0.197(0.371)$ & $0.219(0.346)$ \\
\hline No. of non-H atoms & 3890 & 3447 \\
\hline Macromolecules & 3264 & 3221 \\
\hline Ligands & 117 & 42 \\
\hline Solvent & 509 & 184 \\
\hline Protein residues & 417 & 415 \\
\hline \multicolumn{3}{|l|}{ R.m.s. deviations } \\
\hline Bond length $(\AA)$ & 0.011 & 0.001 \\
\hline Bond angles $\left({ }^{\circ}\right)$ & 1.10 & 0.41 \\
\hline \multicolumn{3}{|l|}{ Ramachandran plot } \\
\hline Favored (\%) & 96.85 & 96.84 \\
\hline Allowed (\%) & 2.91 & 2.92 \\
\hline Outliers (\%) & 0.24 & 0.24 \\
\hline Average $B$ factor $\left(\AA^{2}\right)$ & 26.61 & 44.43 \\
\hline Macromolecules & 24.60 & 44.11 \\
\hline Ligands & 37.61 & 54.76 \\
\hline Solvent & 36.94 & 47.72 \\
\hline
\end{tabular}

444 Statistics for the highest-resolution shell are shown in parentheses. 
bioRxiv preprint doi: https://doi.org/10.1101/2021.04.02.438274; this version posted April 4, 2021. The copyright holder for this preprint (which

was not certified by peer review) is the author/funder, who has granted bioRxiv a license to display the preprint in perpetuity. It is made available under aCC-BY-NC-ND 4.0 International license.

Table 3: Root-mean-square fluctuations (RMSF, in $\AA$ ) of the catalytic residues and RNA-binding residues in the 3 simulated systems.

448 RMSF of $\mathrm{C} \alpha$ atoms were calculated after aligning trajectories to the initial model with respect to C $\alpha$

449 atoms of residues 71-289 (core of the ExoN domain). RMSF of all atoms for each residue is presented in 450 parenthesis. Catalytic residues of ExoN are underlined.

451

\begin{tabular}{|c|c|c|c|}
\hline & nsp14 & nsp14-nsp10 & nsp14-nsp10-RNA \\
\hline D90 (nsp14) & $0.44(0.56)$ & $0.40(0.52)$ & $0.35(0.39)$ \\
\hline E92 (nsp14) & $0.61(1.18)$ & $0.63(1.16)$ & $0.39(0.76)$ \\
\hline E191 (nsp14) & $0.58(0.76)$ & $0.56(0.75)$ & $0.38(0.61)$ \\
\hline H268 (nsp14) & $1.75(2.33)$ & $1.66(2.21)$ & $1.34(2.05)$ \\
\hline D273 (nsp14) & $0.58(0.97)$ & $0.60(0.92)$ & $0.39(0.45)$ \\
\hline K9 (nsp14) & $1.80(2.59)$ & $1.16(1.62)$ & $0.55(0.67)$ \\
\hline K61 (nsp14) & $2.81(3.49)$ & $1.60(2.26)$ & $0.73(1.25)$ \\
\hline K139 (nsp14) & $0.95(1.56)$ & $0.86(1.52)$ & $0.62(1.18)$ \\
\hline A1 (nsp10) & & $4.04(4.12)$ & $0.75(0.82)$ \\
\hline
\end{tabular}


A

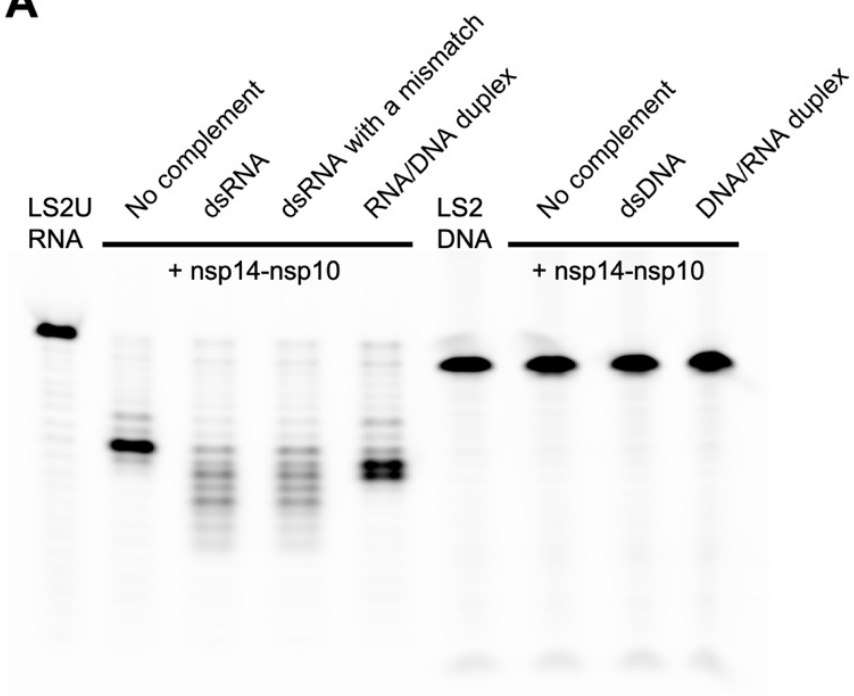

B

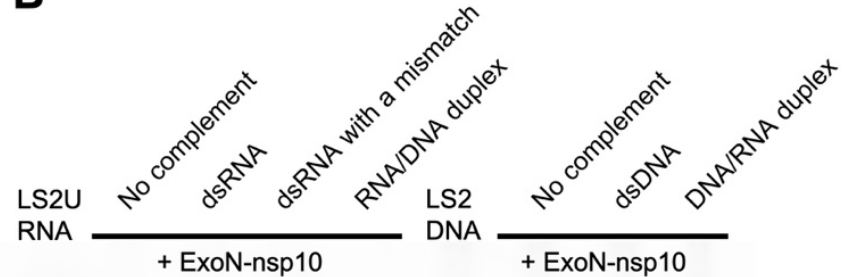

C

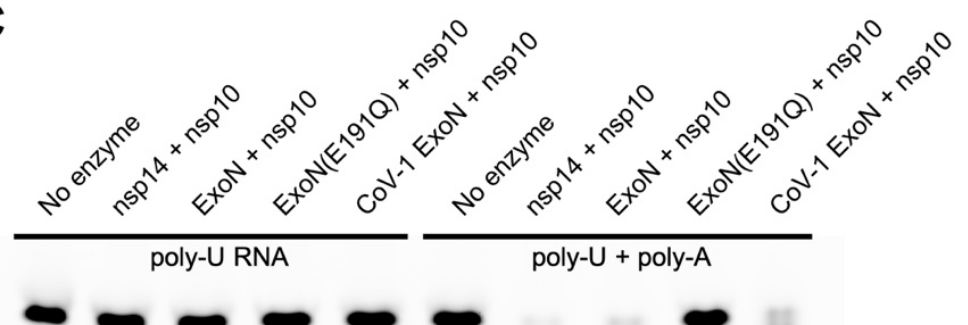

Fig. 1. Biochemical activities of nsp14 or its N-terminal ExoN domain, in complex with nsp10.

456 A, Exonuclease activities of SARS-CoV-2 full-length nsp14-nsp10 complex on various RNA and DNA

457 substrates. B, Exonuclease activities of SARS-CoV-2 ExoN (nsp14 residues 1-289)-nsp10 complex on

458 the same set of RNA and DNA substrates as in (a). C, Exonuclease activities of SARS-CoV-2 full-

459 length nsp14-nsp10, SARS-CoV-2 ExoN-nsp10, and SARS-CoV ExoN-nsp10 complexes on poly-U

460 RNA in the absence (left) or presence (right) of unlabeled poly-A RNA. Please see Table 1 for the

461 substrate sequences. 


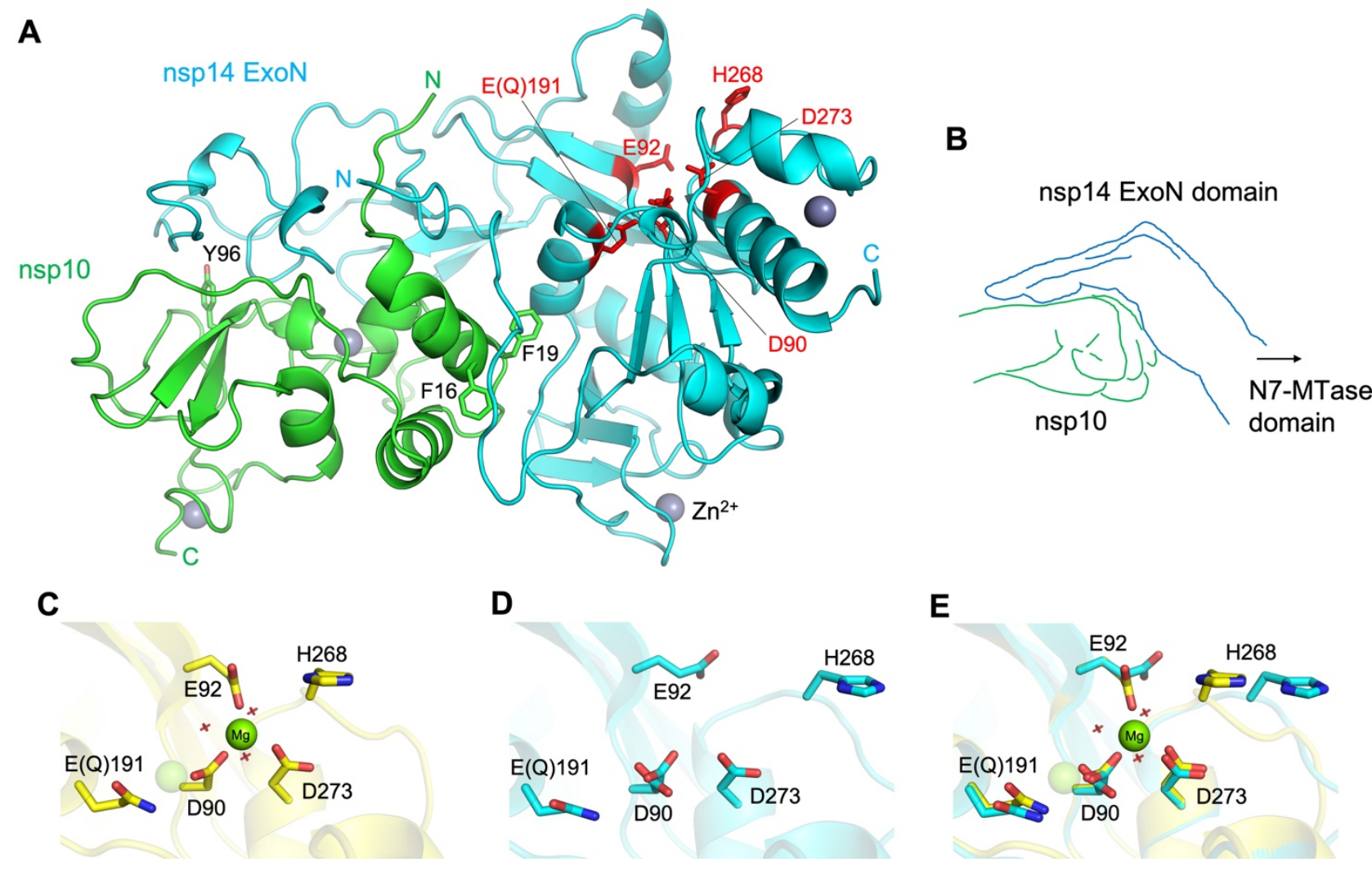

464 Fig. 2. SARS-CoV-2 ExoN-nsp10 structure and its active site flexibility

465 A, Overall structure of nsp14(1-289)-nsp10 complex. The N-terminal ExoN domain of nsp14 is shown 466 in cyan and nsp10 in green. The ExoN active site residues are highlighted as red sticks. Key aromatic 467 residues of nsp10 in the protein-protein interface are also shown as sticks. Gray spheres represent zinc 468 ions. B, A schematic illustration of hand (ExoN) over a fist (nsp10). C, ExoN active site in the presence 469 of $\mathrm{Mg}^{2+}$. The magnesium ion is shown as a solid sphere scaled at half the van der Waals radius. The 470 second $\mathrm{Mg}^{2+}$-binding site, indicated by a transparent sphere, is unoccupied in our structure presumably 471 due to the E191Q mutation. D, $\mathrm{Mg}^{2+}$-free active site as observed in the tartrate-bound crystal. Asp90 472 side chain shows a dual conformation. E, Superposition of $\mathbf{C}$ and $\mathbf{D}$, highlighting the conformational 473 changes upon $\mathrm{Mg}^{2+}$-binding. 
A

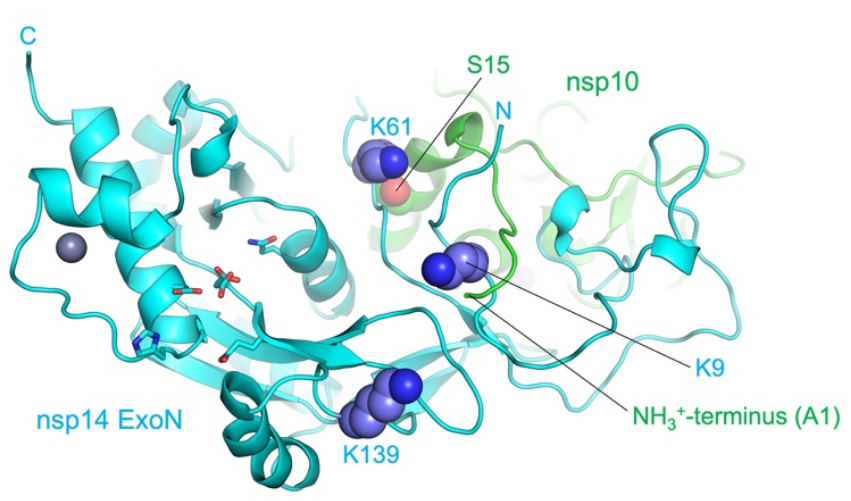

C

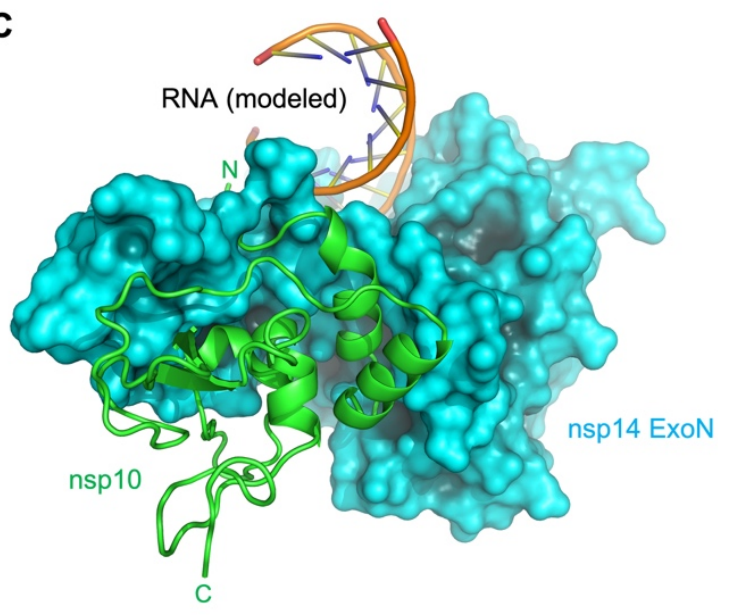

B
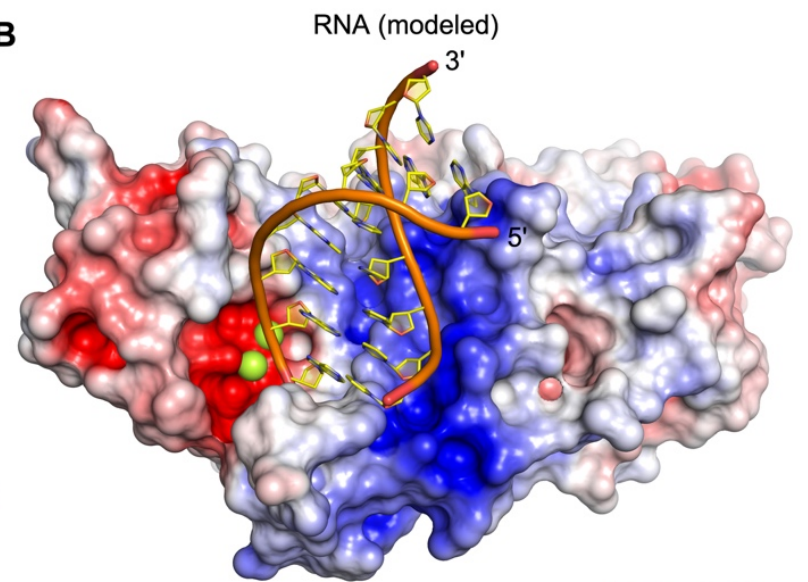

$\bigcup_{45^{\circ}}$

$-5 \square 5 \mathrm{kT} / \mathrm{e}$

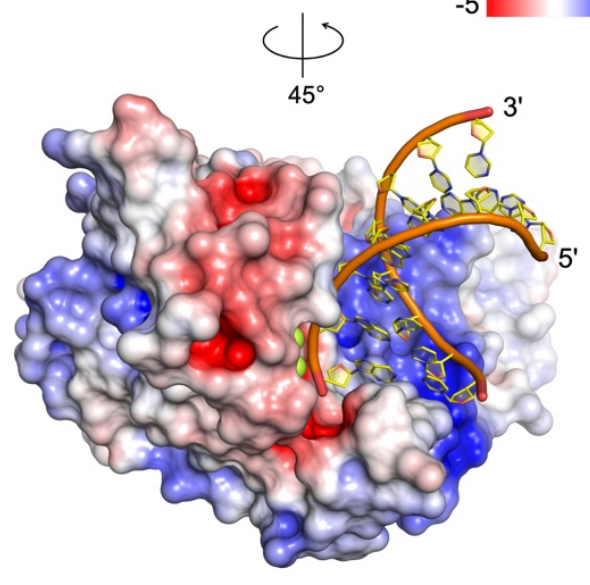

475 Fig. 3. Location of the basic patch and an ExoN-nsp10-RNA complex model

476 A, Locations of the ExoN lysine residues forming the basic patch. Note that Lys9 and Lys61 interact with the N-terminus (Ala1) and Ser15 of nsp10, respectively. B, A hypothetical model of ExoN-nsp10dsRNA complex, viewed from two different orientations. The protein surface is colored according to the electrostatic potential calculated using APBS (46). C,

C, Backside of the ExoN-nsp10-dsRNA model, viewed from the ExoN-nsp10 interface. Nsp10 is shown as green ribbon. 
A

\section{LS2U alone}

No
enzyme WT K9A K61A K139A

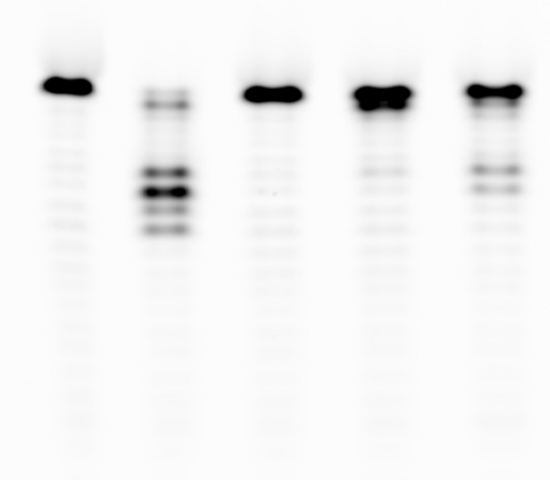

B

dsRNA substrate

LS2U + LS15A_RNA

No
enzyme WT K9A K61A K139A

\section{Fig. 4. Activities of ExoN lysine mutants}

482 Exoribonuclease activities of SARS-CoV-2 ExoN-nsp10 complex and its lysine-to-alanine point mutant

483 derivatives. A, Processing of LS2U RNA without a complementary strand. B, LS2U RNA annealed with

484 the fully complementary LS15A RNA (dsRNA substrate). Please see Table $\mathbf{1}$ for the substrate

485 sequences. 


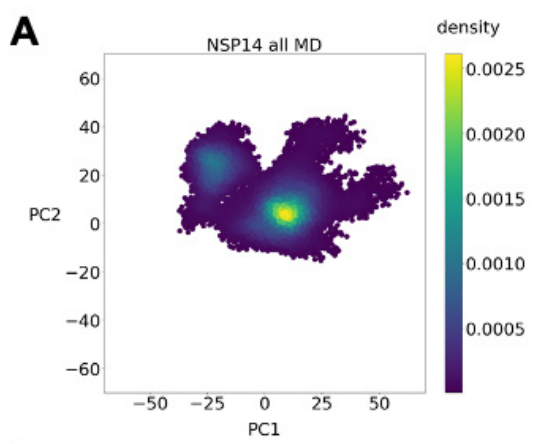

B

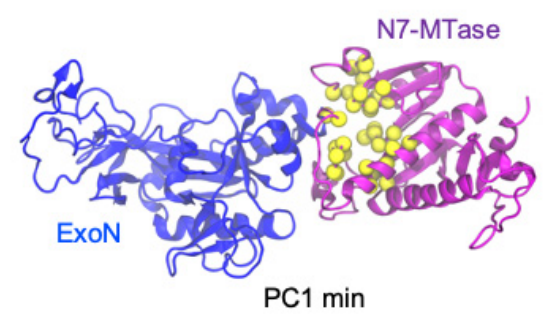

C

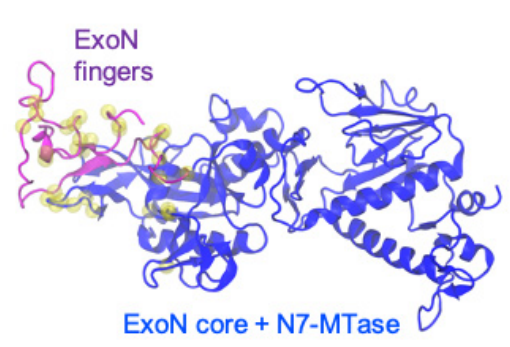

$\mathrm{PC} 2 \mathrm{~min}$

$\mathbf{F}$

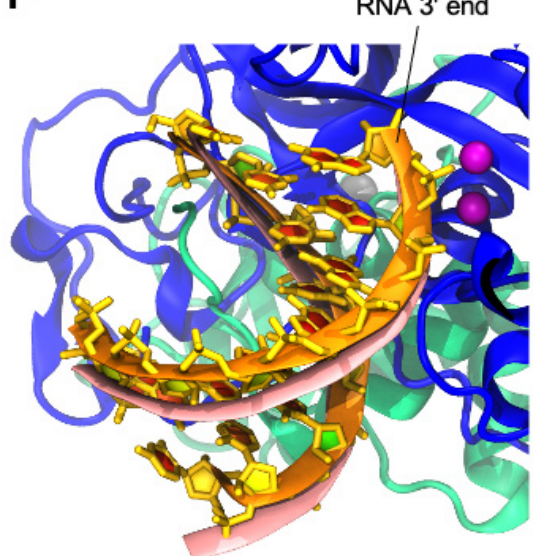

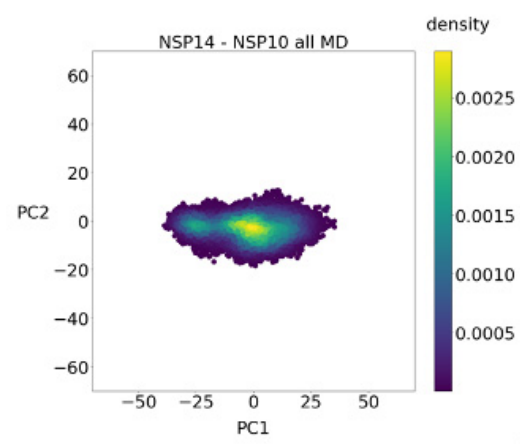
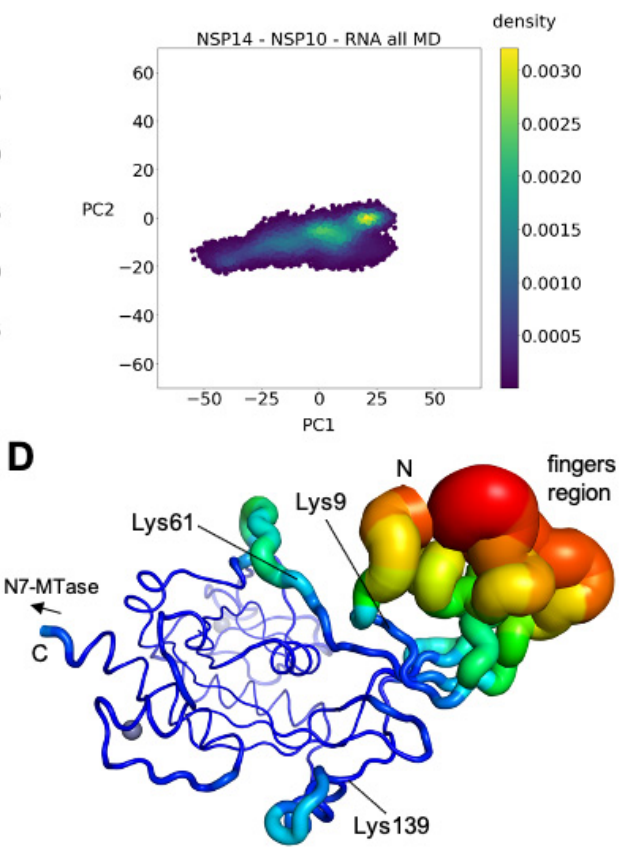

E
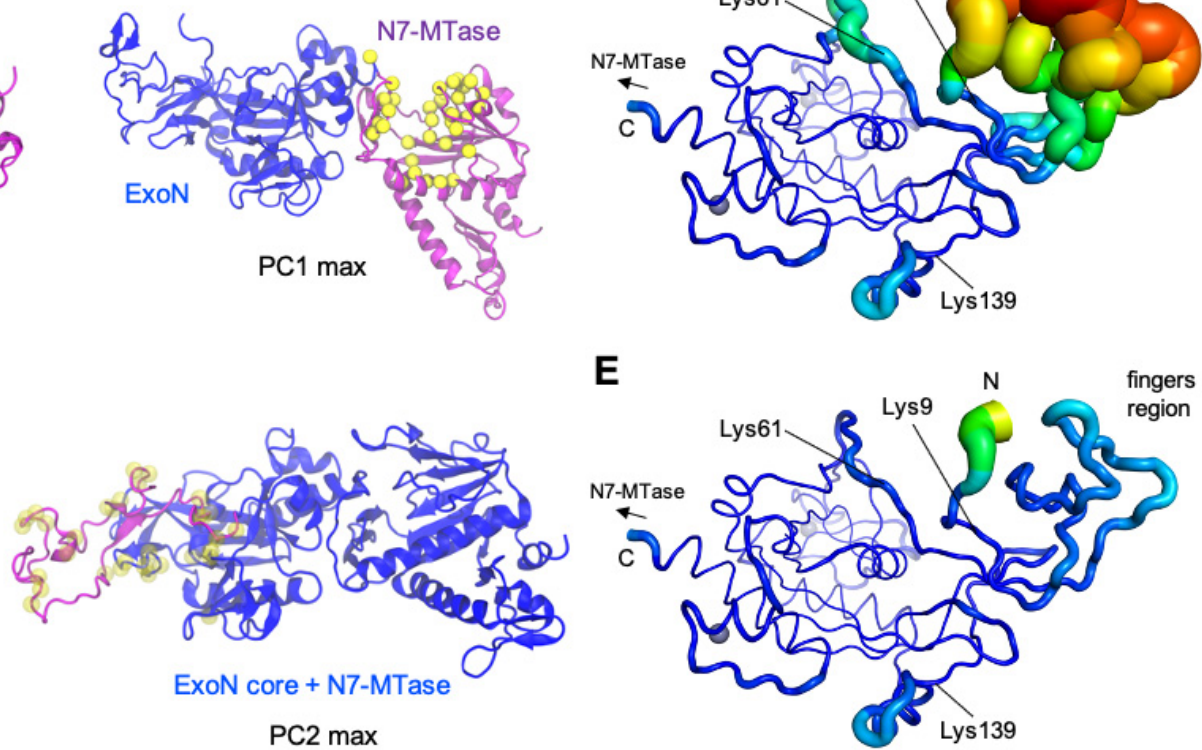

PC2 max

Fig. 5. MD simulations.

G

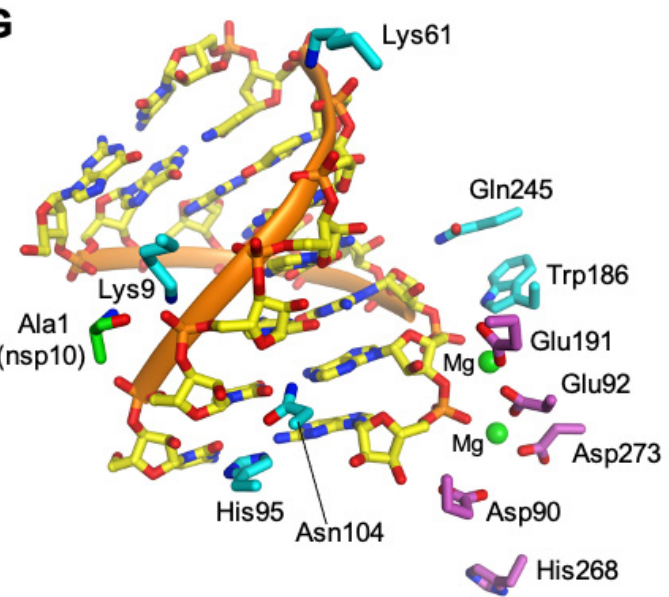

487 A, Principal component analysis depicting differential conformational sampling for the 3 systems in MD

488 simulations. B, Structures that correspond to PC1 minimum and maximum values for the nsp14-alone 489 system. N7-MTase and ExoN domains of nsp14 are depicted in purple and blue ribbons, respectively.

490 Yellow spheres represent the C $\alpha$ atoms of residues that constitute the binding site of SAM and GpppA 
491 substrates of N7-MTase based on homology to SARS-CoV nsp14 N7-MTase crystal structures (PDB

492 ID: 5C8S and 5C8T)(24). C, Structures that correspond to PC2 minimum and maximum values for the 493 nsp14-alone system. N-terminal region (residues 1-71) of nsp14 is depicted in purple ribbons while the 494 rest of nsp14 is depicted in blue ribbons. Transparent yellow spheres represent the C $\alpha$ atoms of nsp14 495 residues that constitute nsp10 binding site. D, ExoN domain in nsp14-alone system with root-mean496 square fluctuations (RMSF) of C $\alpha$ atoms depicted on the structure with varying tube thickness and color 497 (low in blue to high in red). The view is similar to that in Fig. 3A. E, ExoN domain of nsp14-nsp10 498 system with C $\alpha$ RMSF depicted on the structure with varying tube thickness and color. F, RNA after 1 $499 \mu \mathrm{s}$ MD simulation (in orange ribbons) of nsp14-nsp10-RNA system superimposed onto RNA of the 500 starting model (salmon). Nsp14 and nsp10 are depicted as blue and green ribbons, respectively. Dark 501 purple spheres represent two $\mathrm{Mg}$ ions in the active site. G, RNA after $1 \mu$ s MD simulation of the nsp14502 nsp10-RNA system, with nsp14 ExoN domain (cyan) or nsp10 (green) residues making persistent 503 hydrogen-bond or salt bridge interactions with RNA in MD simulations shown as sticks. The active site 504 residues of ExoN are also shown (purple sticks) with two $\mathrm{Mg}^{2+}$ ions as green spheres. 


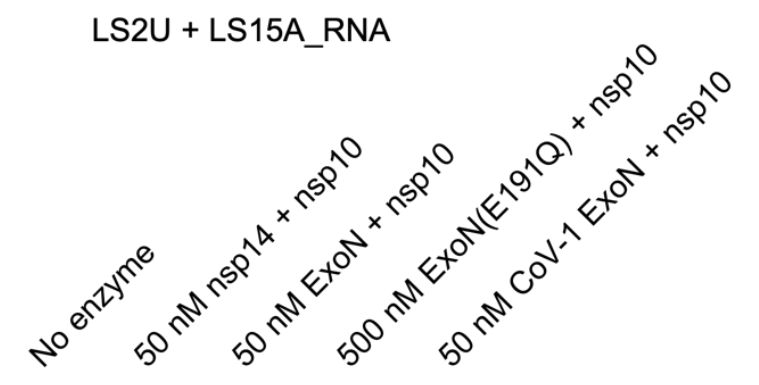

506 Supplementary Fig. 1 | Comparison of exoribonuclease activities

507 Exoribonuclease activities of SARS-CoV-2 nsp14-nsp10, ExoN-nsp10, and SARS-CoV ExoN-nsp10

508 complexes on a double-stranded RNA substrate. The inactive E191Q mutant enzyme was tested at a 10 509 times higher protein concentration. 

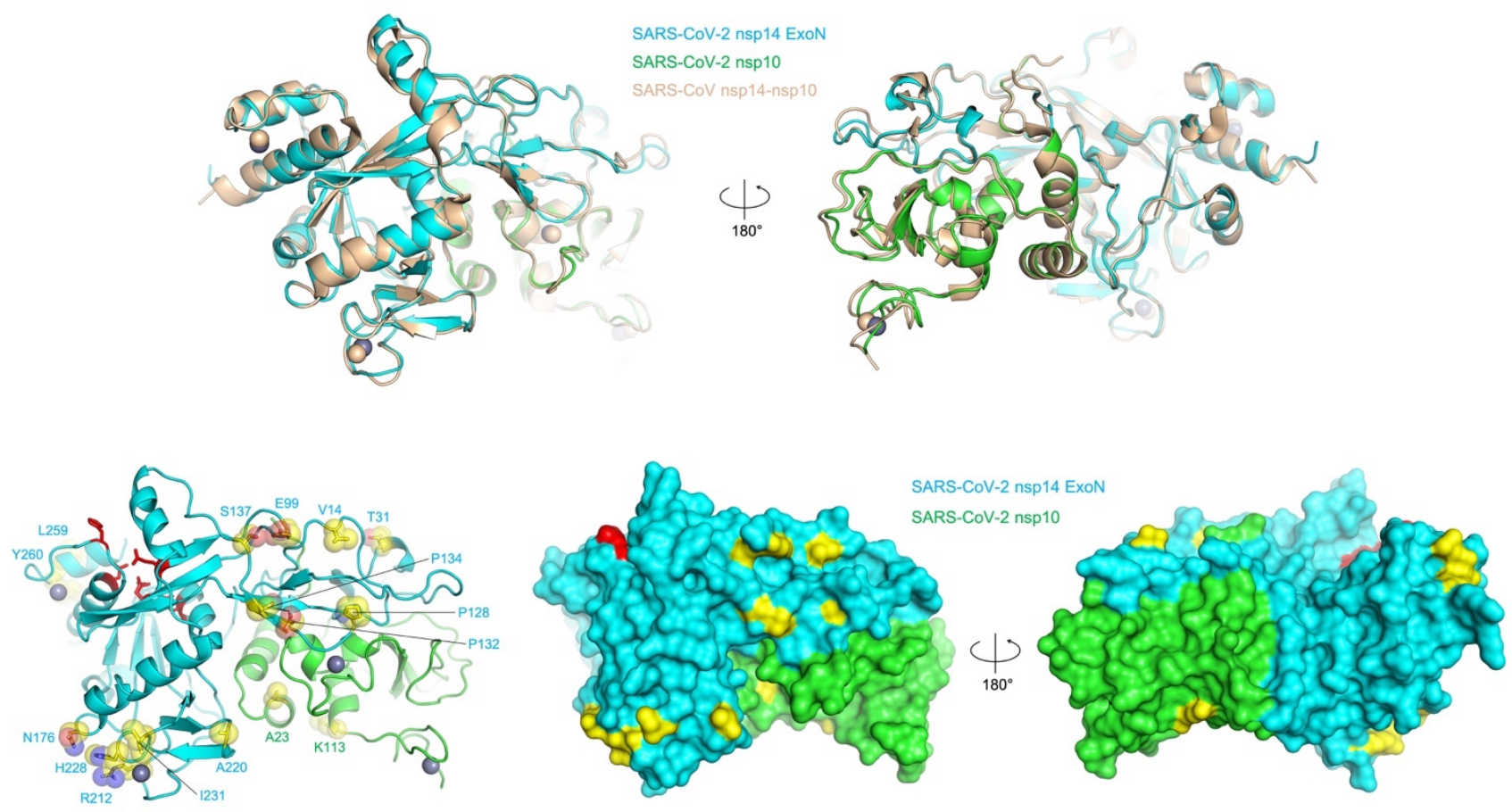

Non-identical residues between SARS-CoV and SARS-CoV-2 are highlighted in yellow.

\section{Supplementary Fig. 2 | SARS-CoV vs. SARS-CoV-2 ExoN-nsp10 structure comparison}

513 Top, A superposition between SARS-CoV (PDB ID: 5C8T) (24) and SARS-CoV-2 (this study) ExoN-

514 nsp10 structures. Bottom, Difference in the amino acid sequence between SARS-CoV and SARS-CoV-

5152 mapped on the ExoN-nsp10 structure and highlighted in yellow. The active site residues are shown in red. 

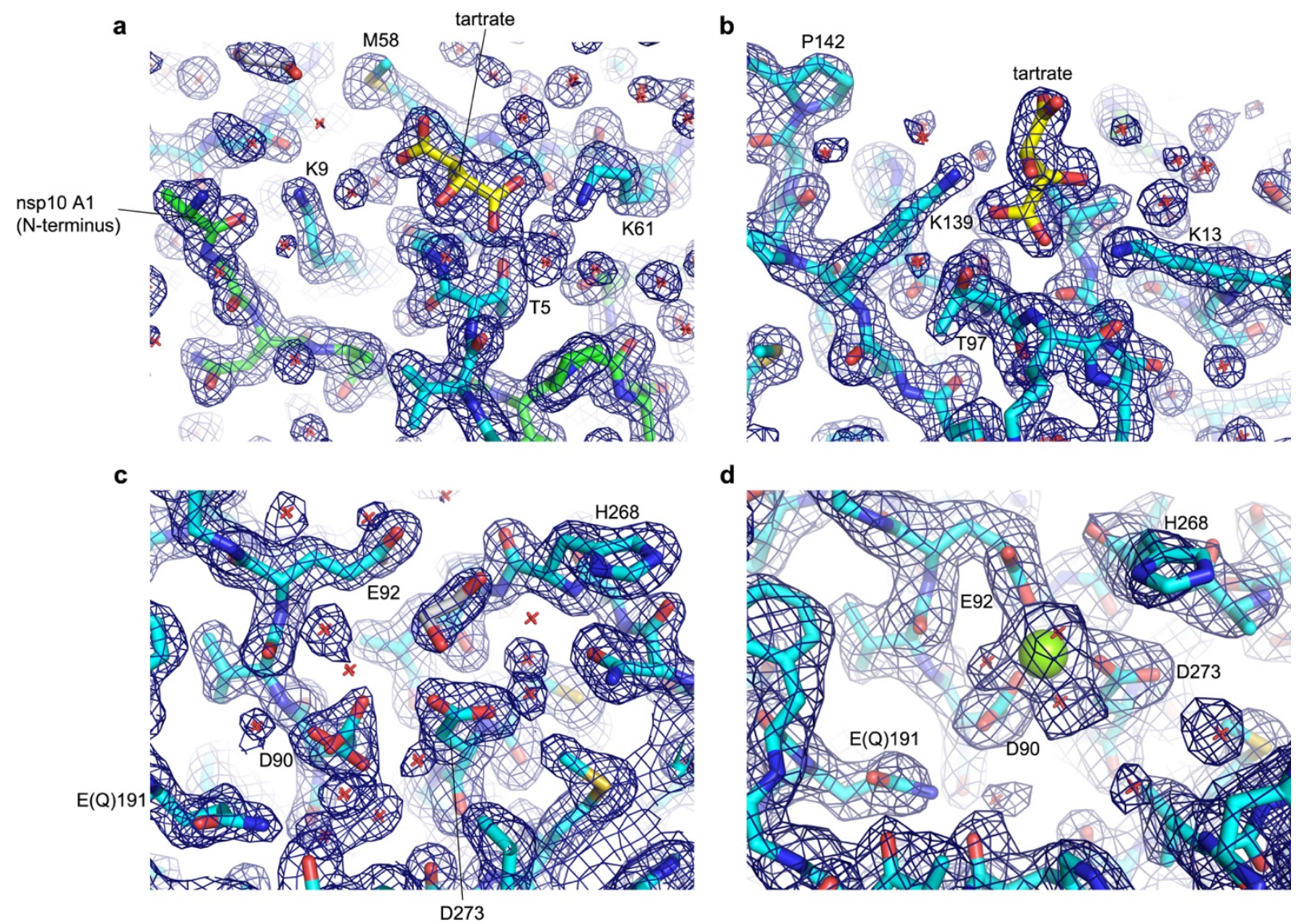

d

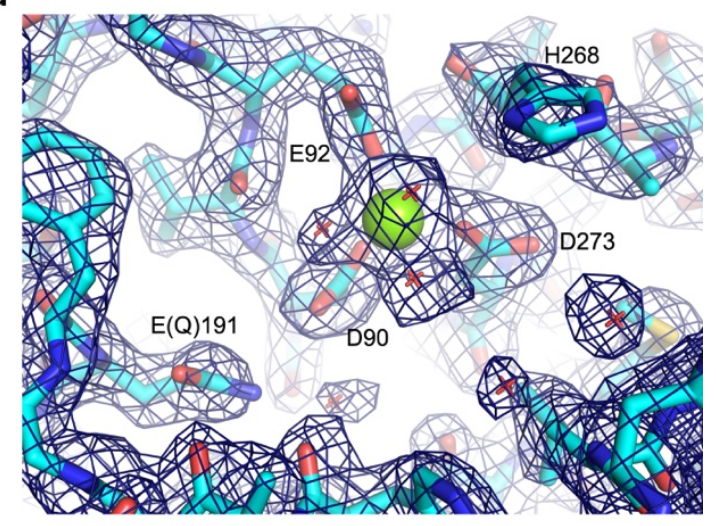

\section{Supplementary Fig. 3 | Electron density maps}

$5182 \mathrm{mFo}-\mathrm{DFc}$ map contoured at $1.0 \sigma$ is shown for the higher resolution $(1.64 \AA)$ tartrate-bound structure in

519 a-c, and for the lower resolution $(2.10 \AA) \mathrm{Mg}^{2+}$-bound structure in $\mathbf{d}$.

520 a, Region including Lys9 and Lys61 of nsp14/ExoN and the N-terminus of nsp10 (The crystallized

521 protein has additional methionine residue on the N-terminus, which is likely to be disordered) with a

522 bound tartrate molecule.

523 b, Region including Lys139 and Lys13 with a tartrate molecule bound between the two lysine side

524 chains.

525 c, $\mathrm{Mg}^{2+}$-free active site. An ethylene glycol molecule used as the cryo-protectant was observed.

526 d, $\mathrm{Mg}^{2+}$-bound active site. 


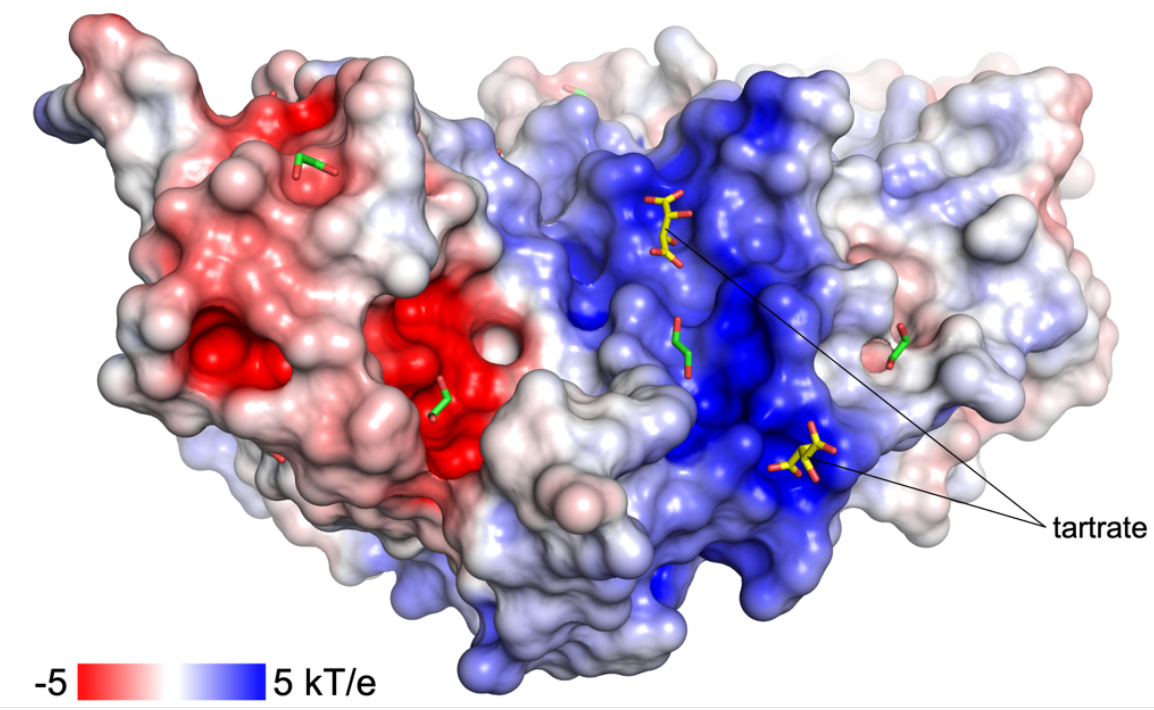

527 Supplementary Fig. 4 | Tartrate ions bound on the basic patch of ExoN-nsp10 complex

528 Electrostatic surface potential of ExoN-nsp10 with tartrate or ethylene glycol bound on the protein

529 surface.

530

531
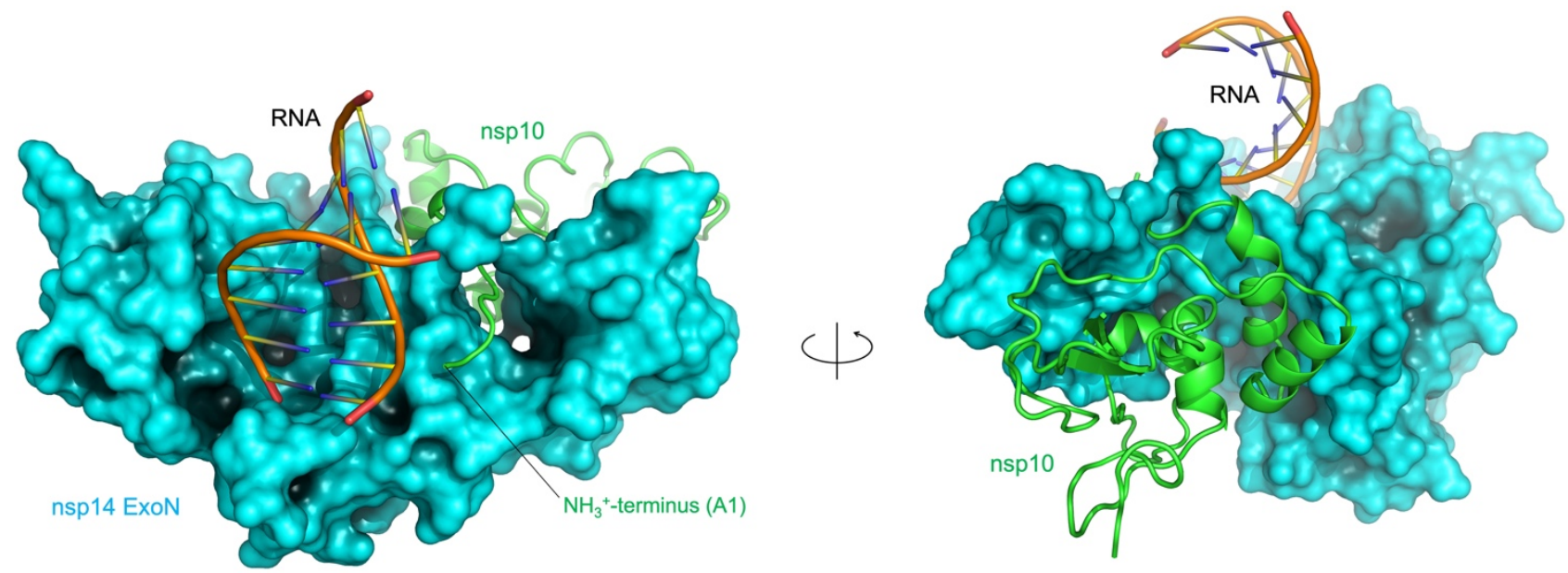

533 Supplementary Fig. 5 | ExoN-nsp10-RNA complex model (an additional view)

534 The image on the right is same as Fig. 3C. 


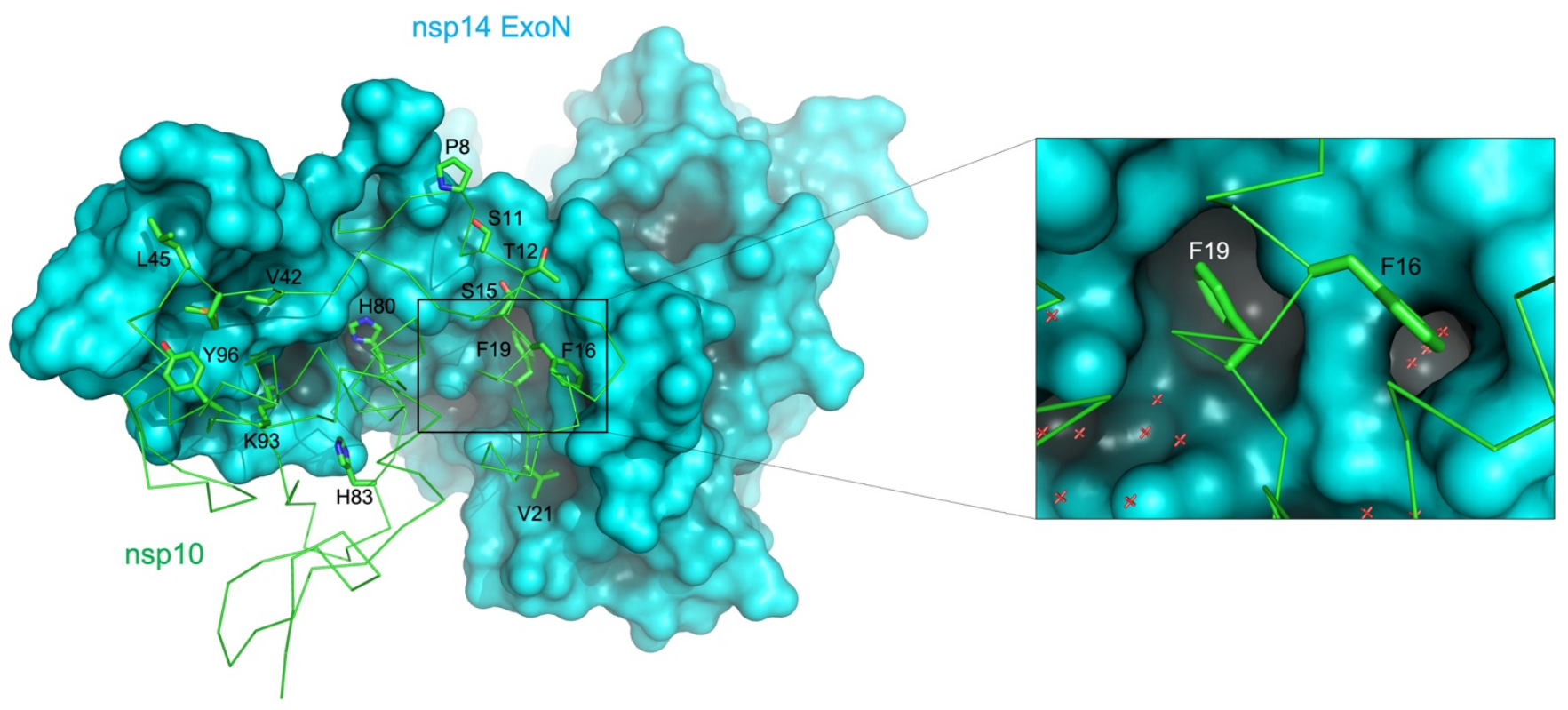

539 Supplementary Fig. 6 | ExoN-nsp10 interface

540 ExoN is shown in solid surface and nsp10 in wire-frame representations, respectively. Some (not all) of

541 the nsp10 side chains involved in the protein-protein interaction are shown as sticks. A zoomed view of

542 the hydrophobic pocket that accommodates Phe16 and Phe19 of nsp10 is shown on the right. Red

543 crosshairs represent water molecules. 

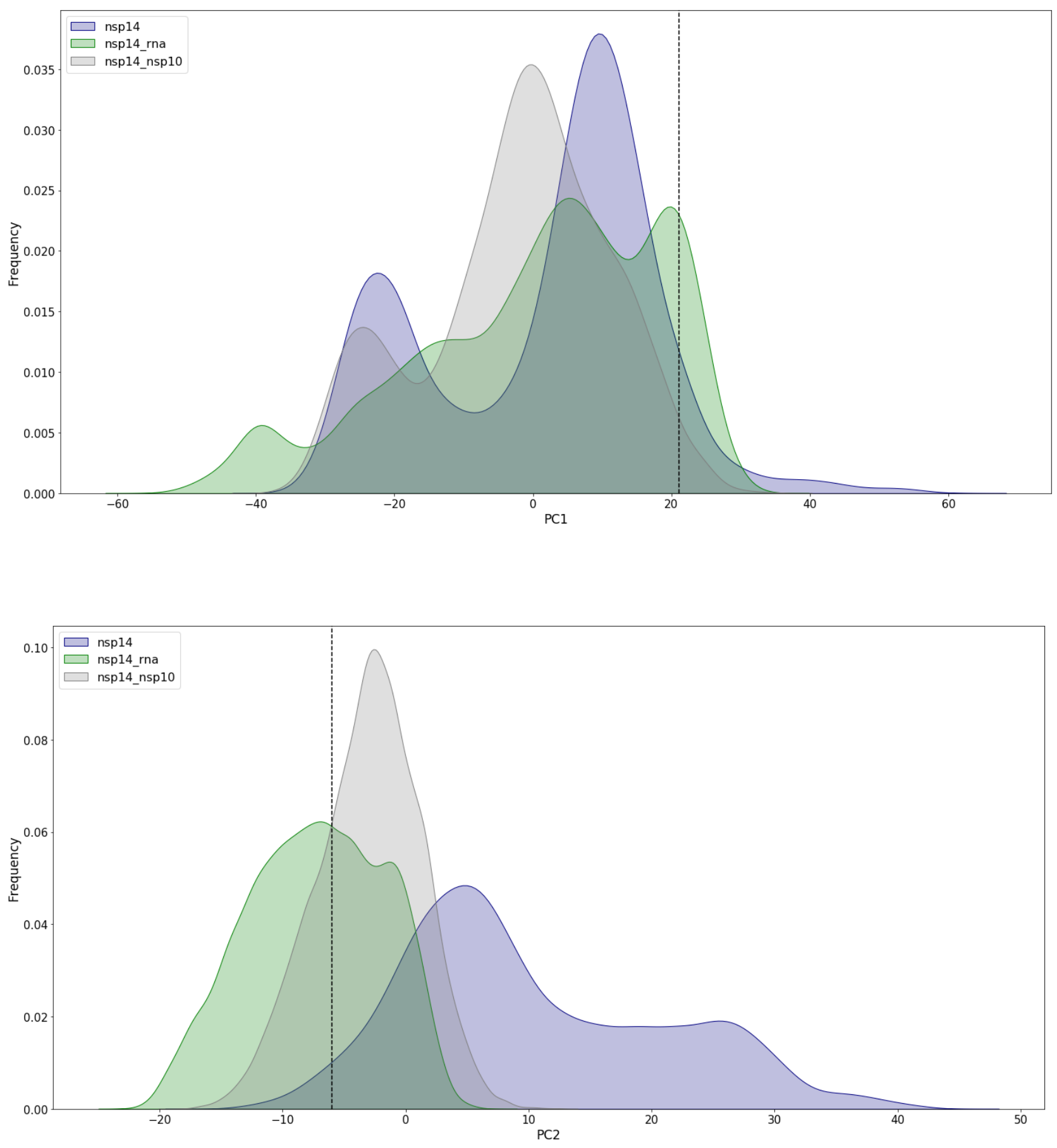

549 Supplementary Fig. 7 | Distribution of principal components 1 and 2 (PC1 and PC2) observed in

550 MD simulations. The dashed line in each plot indicates the value calculated for the starting structure. 

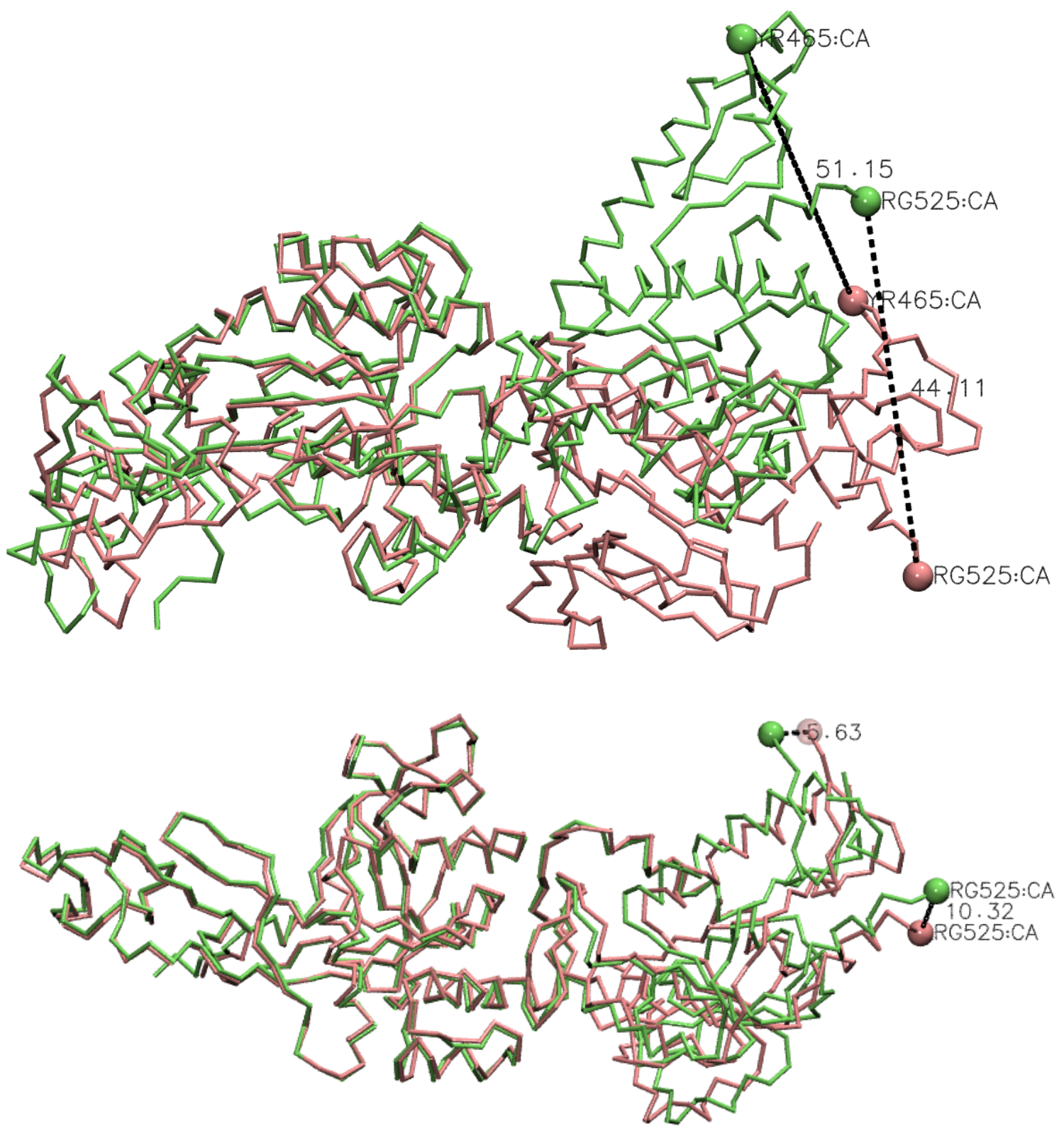

Supplementary Fig. 8 | Conformational flexibility of full-length nsp14.

a, Superposition of the structures that correspond to PC1 minimum and PC1 maximum values in MD simulations based on the ExoN domain, showing large displacement of the N7-MTase domain (This conformational change is also shown in Supplementary animation 1). b, Similar conformational variability, albeit with a smaller magnitude, observed between chains A and B of SARS-CoV nsp14 
a
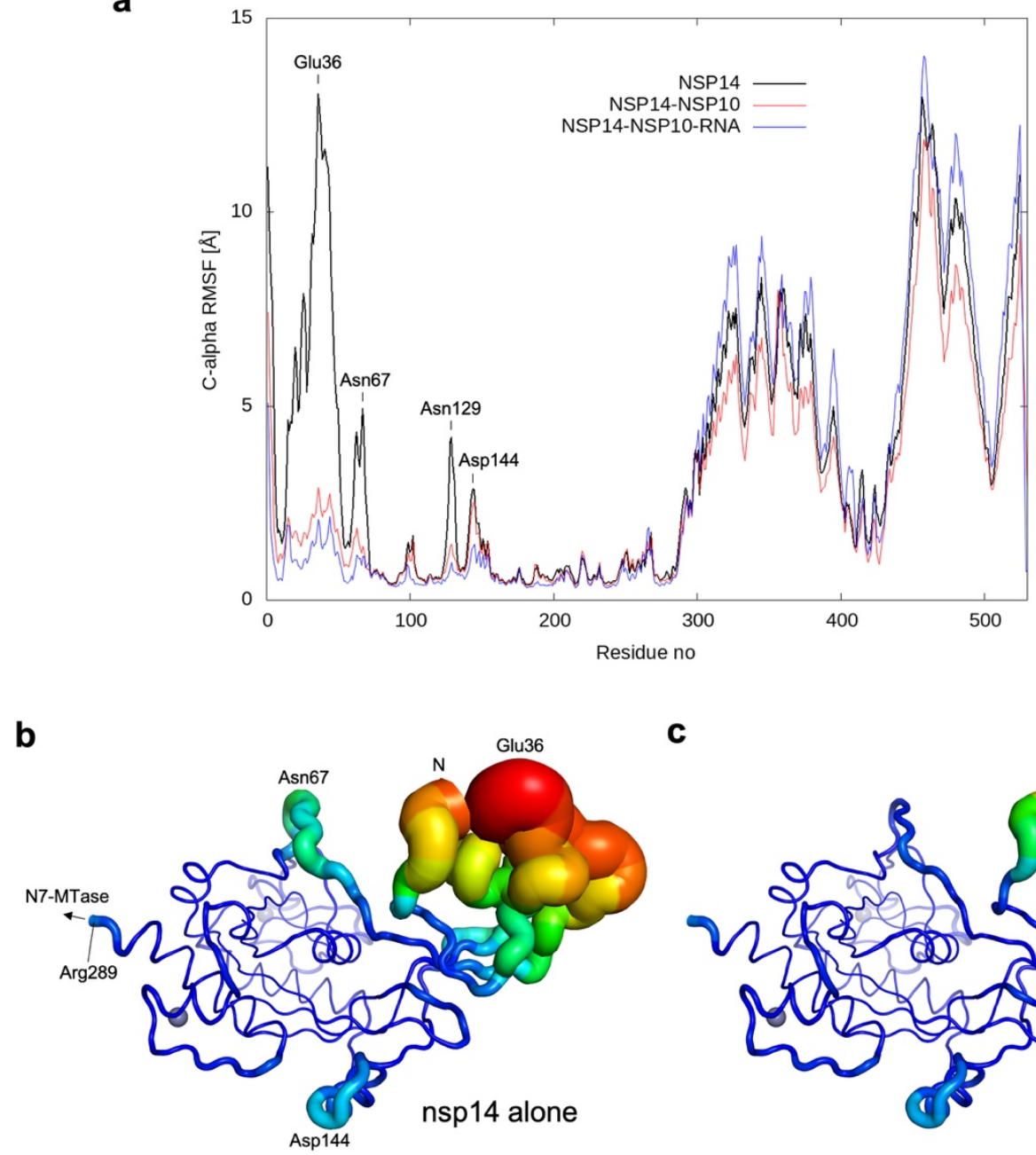

C

d

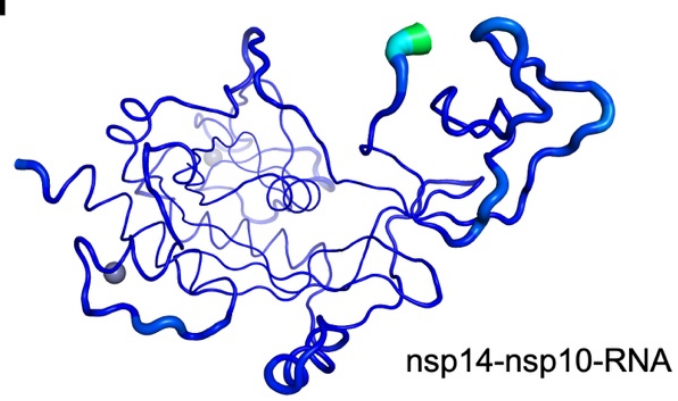

Supplementary Fig. 9 | Internal dynamics of ExoN domain observed in MD simulations.

579 a, Root-mean-square fluctuations (RMSF) for nsp14 C $\alpha$ atoms in MD simulations of the three systems

580 after aligning their trajectories to the starting structure with respect to $\mathrm{C} \alpha$ atoms of nsp14 residues 71-

581 289. b-d, RMSF for nsp14 alone (b), nsp14-nsp10 (c), and nsp14-nsp10-RNA (d), depicted by tube

582 thickness and color. Panels $\mathbf{b}$ and $\mathbf{c}$ are same as Fig. $5 \mathbf{D}$ and $\mathbf{E}$, respectively, and panels b-d correspond 

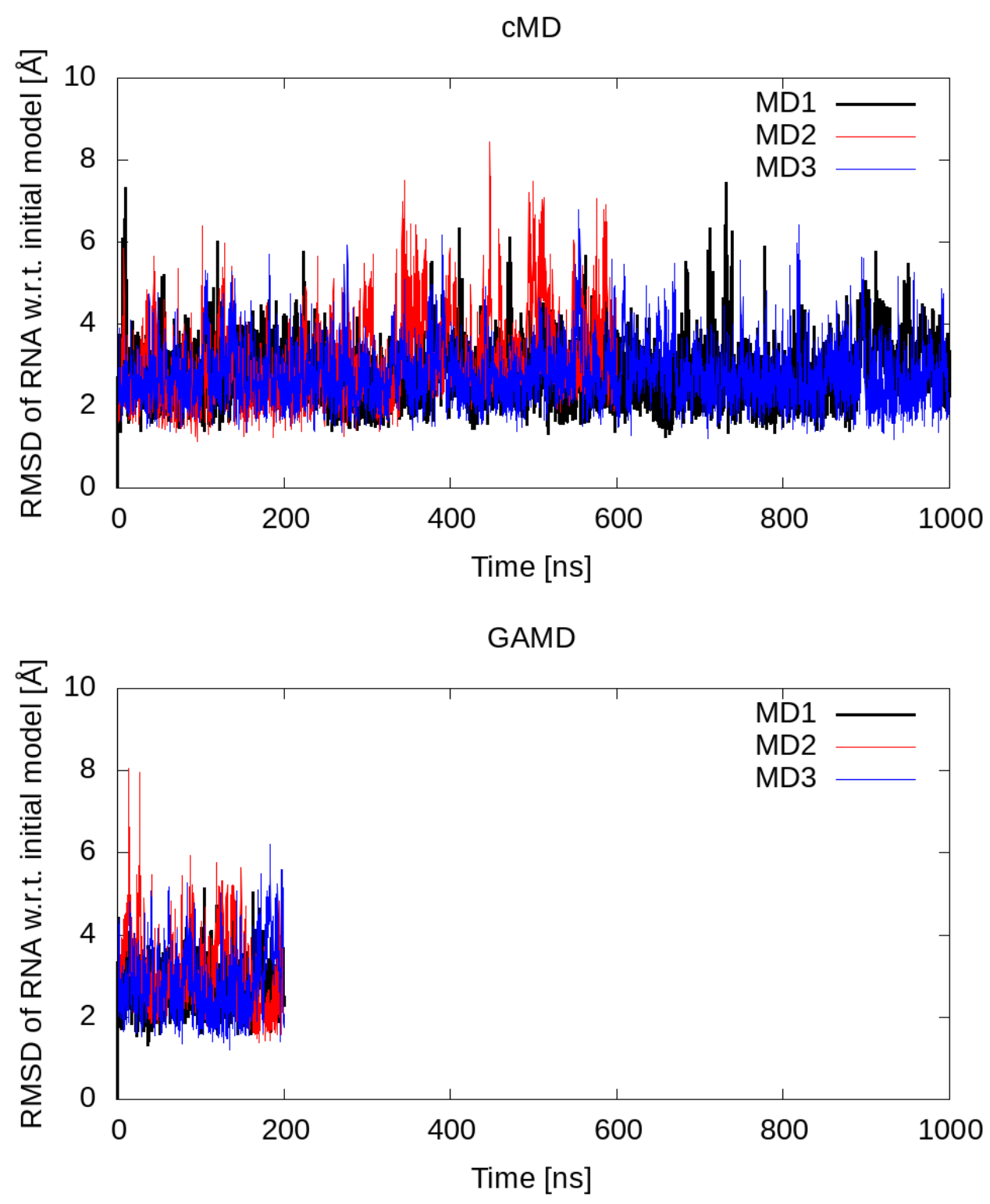

586 Supplementary Fig. 10 | Stability of RNA in MD simulations.

587 Root-mean-square deviation (RMSD) of RNA atoms calculated for conventional and Gaussian-

588 accelerated MD (cMD and GAMD) simulations after aligning the trajectories with respect to C $\alpha$ atoms 589 of nsp14 residues 71-289. 


\section{a}

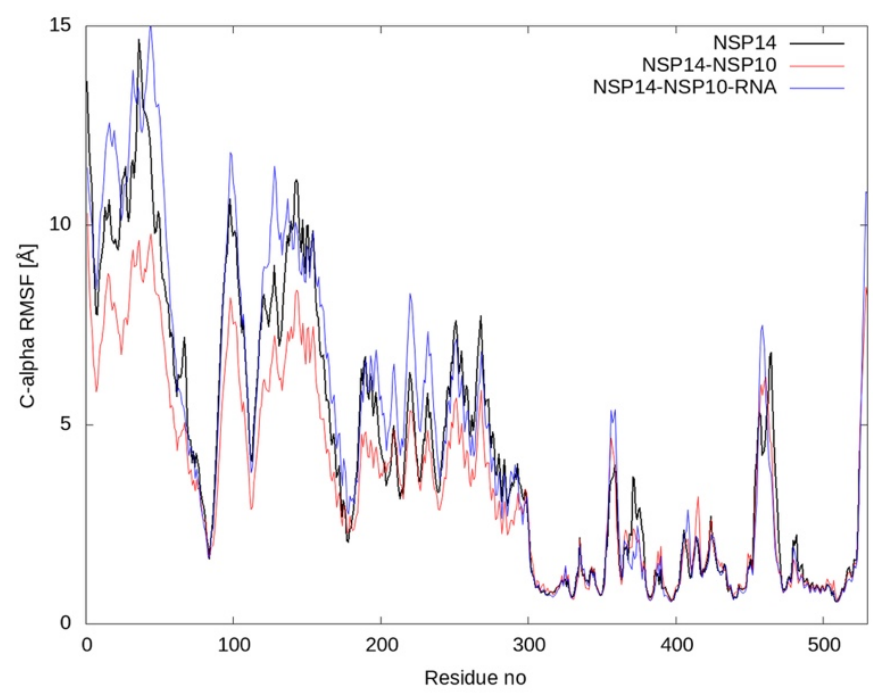

b

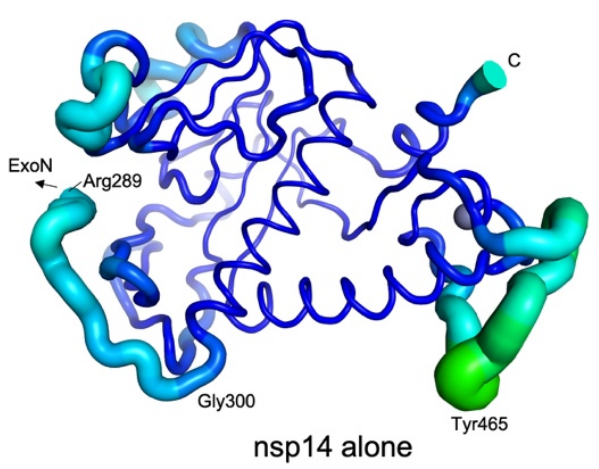

C

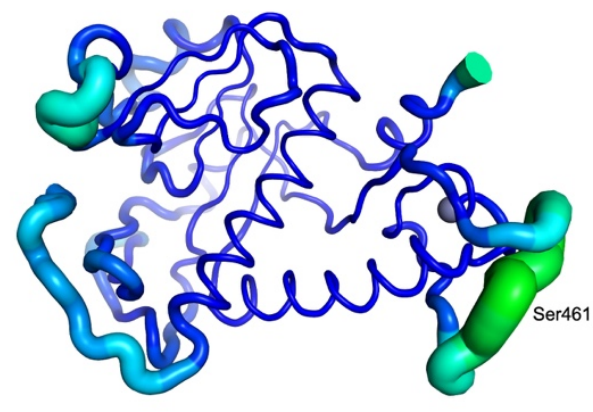

nsp14-nsp10

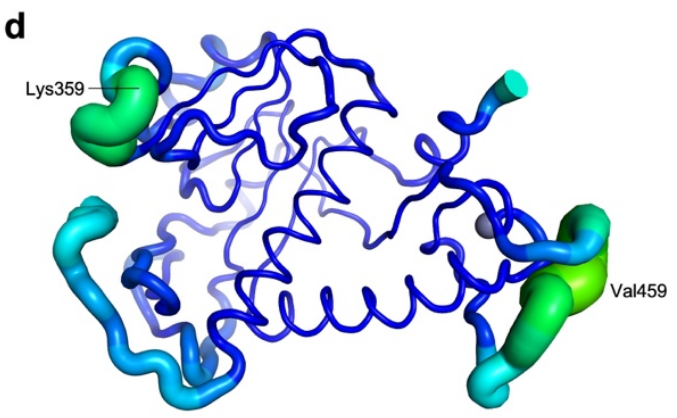

nsp14-nsp10-RNA

591 Supplementary Fig. 11 | Internal dynamics of N7-MTase domain observed in MD simulations.

592 a, Root-mean-square fluctuations (RMSF) for nsp14 C $\alpha$ atoms in MD simulations of the three systems

593 after aligning their trajectories to the starting structure with respect to $\mathrm{C} \alpha$ atoms of nsp14 residues 300-

594 525. b-d, RMSF for nsp14 alone (b), nsp14-nsp10 (c), and nsp14-nsp10-RNA (d), depicted by tube 595 thickness and color. 
bioRxiv preprint doi: https://doi.org/10.1101/2021.04.02.438274; this version posted April 4, 2021. The copyright holder for this preprint (which was not certified by peer review) is the author/funder, who has granted bioRxiv a license to display the preprint in perpetuity. It is made available under aCC-BY-NC-ND 4.0 International license.

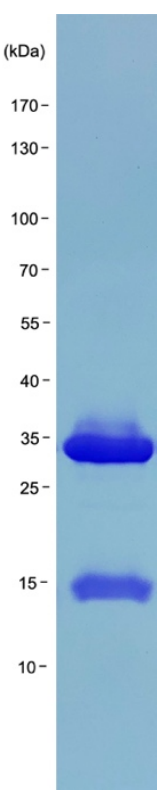

600 Supplementary Fig. 12 | SDS-PAGE of purified SARS-CoV-2 ExoN(E191Q)-nsp10 complex.

601 This protein complex was used in the crystallographic studies. 

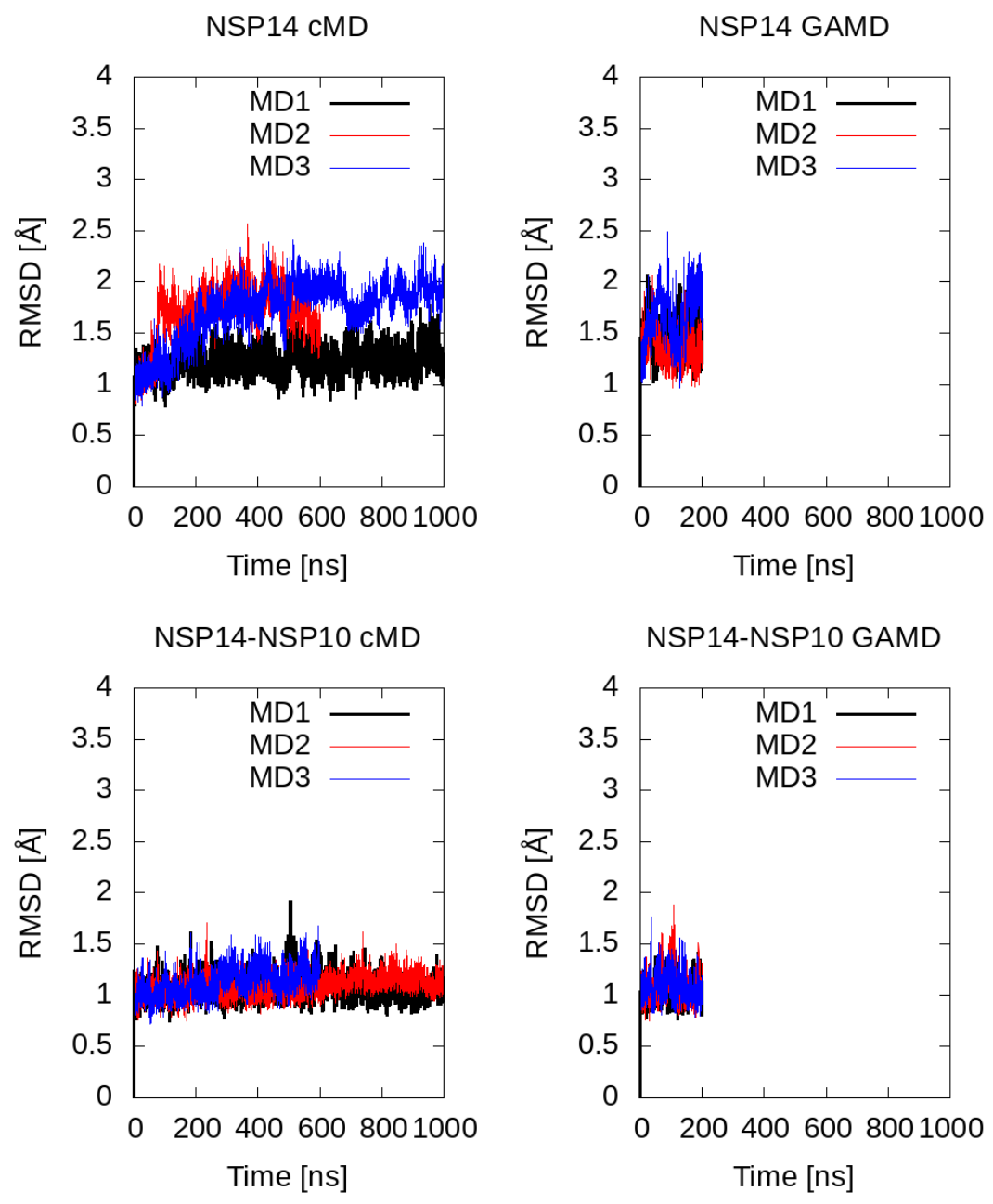

602
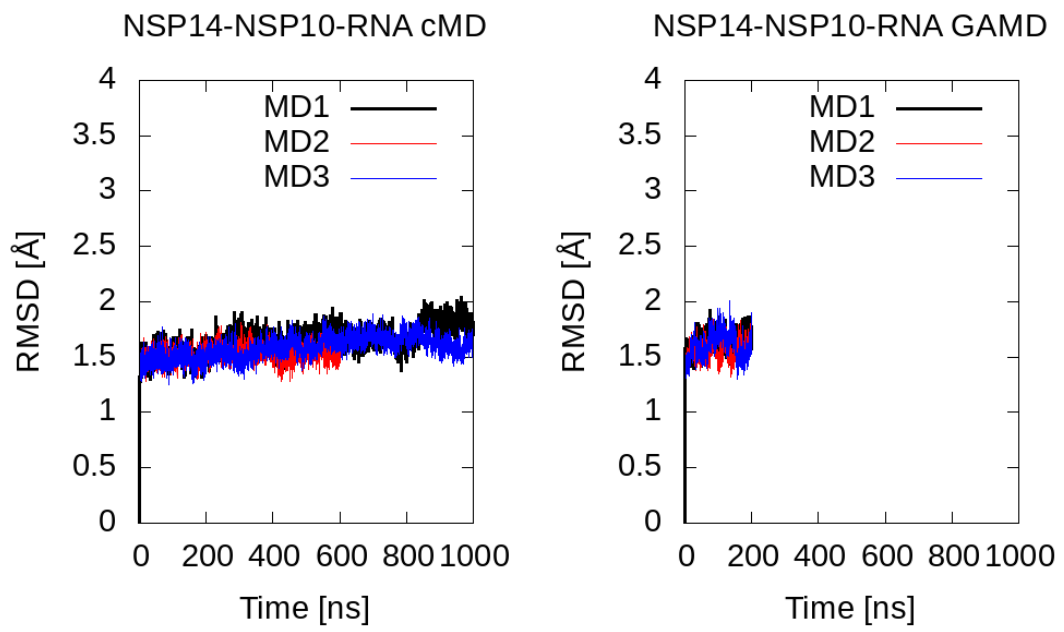

603 Supplementary Fig. 13 | Stability of MD simulations.

604 Root-mean-square deviation (RMSD) of the nsp14 ExoN domain C $\alpha$ atoms (residues 71-289) with

605 respect to the initial model throughout MD simulations. 